\title{
Methane, ammonia, and their irradiation products at the surface of an intermediate-size KBO? A portrait of Plutino (90482) Orcus
}

\author{
A. Delsanti ${ }^{1,2}$, F. Merlin ${ }^{3}$, A. Guilbert-Lepoutre ${ }^{4}$, J. Bauer ${ }^{5}$, B. Yang ${ }^{6}$, and K. J. Meech ${ }^{6}$ \\ 1 Laboratoire d'Astrophysique de Marseille, Université de Provence, CNRS, 38 rue Frédéric Joliot-Curie, 13388 Marseille Cedex 13, \\ France \\ e-mail: Audrey.Delsanti@oamp.fr \\ 2 Observatoire de Paris, Site de Meudon, 5 place Jules Janssen, 92190 Meudon, France \\ e-mail: Audrey.Delsanti@obspm.fr \\ 3 University of Maryland, Department of Astronomy, College Park MD 20742, USA \\ e-mail: merlin@astro.umd.edu \\ 4 UCLA, Earth and Space Sciences department, 595 Charles E. Young Drive East, Los Angeles CA 90095, USA \\ e-mail: aguilbert@ucla.edu \\ 5 Jet Propulsion Laboratory, M/S 183-501, 4800 Oak Grove Drive, Pasadena, CA 91109, USA \\ e-mail: bauer@scn.jpl.nasa.gov \\ 6 NASA Astrobiology Institute at Manoa, Institute for Astronomy, 2680 Woodlawn Drive, Honolulu, Hawaii 96822-1839, USA \\ e-mail: [yangbin;meech]@ifa.hawaii.edu
}

Received 20 February 2010 / Accepted 31 May 2010

\section{ABSTRACT}

\begin{abstract}
Orcus is an intermediate-size $1000 \mathrm{~km}$-scale Kuiper belt object (KBO) in 3:2 mean-motion resonance with Neptune, in an orbit very similar to that of Pluto. It has a water-ice dominated surface with solar-like visible colors. We present visible and near-infrared photometry and spectroscopy obtained with the Keck $10 \mathrm{~m}$-telescope (optical) and the Gemini $8 \mathrm{~m}$-telescope (near-infrared). We confirm the unambiguous detection of crystalline water ice as well as absorption in the $2.2 \mu \mathrm{m}$ region. These spectral properties are close to those observed for Pluto's larger satellite Charon, and for Plutino (208996) 2003 AZ $_{84}$. Both in the visible and near-infrared Orcus' spectral properties appear to be homogeneous over time (and probably rotation) at the resolution available. From Hapke radiative transfer models involving intimate mixtures of various ices we find for the first time that ammonium $\left(\mathrm{NH}_{4}^{+}\right)$and traces of ethane $\left(\mathrm{C}_{2} \mathrm{H}_{6}\right)$, which are most probably solar irradiation products of ammonia and methane, and a mixture of methane and ammonia (diluted or not) are the best candidates to improve the description of the data with respect to a simple water ice mixture (Haumea type surface). The possible more subtle structure of the $2.2 \mu \mathrm{m}$ band(s) should be investigated thoroughly in the future for Orcus and other intermediate size Plutinos to better understand the methane and ammonia chemistry at work, if any. We investigated the thermal history of Orcus with a new 3D thermal evolution model. Simulations over $4.5 \times 10^{9} \mathrm{yr}$ with an input $10 \%$ porosity, bulk composition of $23 \%$ amorphous water ice and $77 \%$ dust (mass fraction), and cold accretion show that even with the action of long-lived radiogenic elements only, Orcus should have a melted core and most probably suffered a cryovolcanic event in its history which brought large amounts of crystalline ice to the surface. The presence of ammonia in the interior would strengthen the melting process. A surface layer of a few hundred meters to a few tens of kilometers of amorphous water ice survives, while most of the remaining volume underneath contains crystalline ice. The crystalline water ice possibly brought to the surface by a past cryovolcanic event should still be detectable after several billion years despite the irradiation effects, as demonstrated by recent laboratory experiments.
\end{abstract}

Key words. Kuiper belt objects: individual: (90482) Orcus - methods: observational - methods: numerical - techniques: photometric - techniques: spectroscopic - radiative transfer

\section{Introduction}

In the past decade, several large Kuiper belt objects (KBOs) have been discovered, unveiling the upper part of the size distribution of these outer Solar System minor bodies. Eris, discovered in 2003 (Brown et al. 2005), is the largest object known to date, and its diameter in the $2500-3000 \mathrm{~km}$ range, exceeding that of Pluto, motivated the discussion and the creation of a new class of objects in 2006: the dwarf planets. Several other large objects were discovered since 2003 (Sedna, Makemake, Haumea, etc.) and focused the interest of scientists: the largest of them soon revealed a volatile-rich surface, dominated by methane, as for Pluto.
Orcus was discovered in 2004 as one of the largest known KBOs (Brown et al. 2004) and is a peculiar object from several aspects. It revolves around the Sun in a Pluto-like orbit, in 3:2 mean motion resonance with Neptune (with a semi-major axis of $39.16 \mathrm{AU}$, an inclination of $20.6^{\circ}$, an eccentricity 0.23 , a perihelion of $30.26 \mathrm{AU}$ and an aphelion at $48.06 \mathrm{AU}$ ). It is currently outbound, very close to aphelion. With its $\sim 950 \mathrm{~km}$ diameter (Stansberry et al. 2008; Brown et al. 2010), it is now part of the intermediate-size objects, and is probably in a transition regime with respect to volatile surface content. Indeed, smaller KBOs with mostly featureless near-IR reflectance spectra seem to be totally depleted in volatiles (see Guilbert et al. 2009, and references therein), while the largest KBOs show 
Table 1. Summarized properties of the Orcus/Vanth binary system.

\begin{tabular}{lllc}
\hline \hline & Parameter & Value & Reference \\
\hline \multirow{2}{*}{ System } & Angular separation & $0.26^{\prime \prime}$ & $(1)$ \\
& Total mass & $6.3 \times 10^{20} \mathrm{~kg}$ & $(2)$ \\
\hline \multirow{2}{*}{ Orbit } & Semi-major axis & $8980 \pm 20 \mathrm{~km}$ & $(2)$ \\
& Period & $9.5 \mathrm{~d}$ & $(2)$ \\
& Inclination & $90^{\circ}$ or $306^{\circ}$ & $(2)$ \\
\hline \multirow{2}{*}{ Satellite } & Brightness Frac. & $8 \%$ & $(3)$ \\
& Mass ratio & $0.5-0.03^{a}$ & $(2)$ \\
& Diameter & $280-640 \mathrm{~km}^{b}$ & $(2)$ \\
\hline
\end{tabular}

Notes. ${ }^{(a)}$ Assuming an albedo ratio of resp. .5 and .1 and equal densities. ${ }^{(b)}$ Assuming an albedo ratio of resp. 1. and .5 and equal densities.

References. References are: (1) Noll et al. (2008), (2) Brown et al. (2010), (3): Brown (2008).

evidence for the presence of volatile ices such as methane and sometimes molecular nitrogen (like Eris, Sedna and Makemake, cf. Merlin et al. 2009; Barucci et al. 2005; Brown et al. 2007a, and references therein). A simple model of atmospheric escape of volatiles dedicated to the Kuiper belt region was computed by Schaller \& Brown (2007b) and showed that the objects that are too small and too hot will most probably lose their pristine volatile surface inventory (such as $\mathrm{CO}, \mathrm{CH}_{4}$ and $\mathrm{N}_{2}$ ) over the age of the Solar System, while large and cold objects could have retained these volatiles to the present day. Objects in an intermediate regime could have lost some volatiles but retained others, following the different loss rates at work for the various species. According to this model, Orcus should have lost all its volatile content. However, traces of methane (to be confirmed) could explain the near-infrared spectrum of Orcus (Barucci et al. 2008). This object can therefore provide key constraints on the current surface volatile inventory of intermediate-size KBOs.

So far, Orcus' rotation properties are not uniquely defined: 0.03 to 0.18 mag amplitude variations are measured over a period ranging from $\sim 7$ to $21 \mathrm{~h}$ (Duffard et al. 2009, and references therein), although Sheppard (2007) finds no lightcurve variations within the photometric uncertainties of his measurements. Such a flat lightcurve could be diagnostic of a very slow rotation rate, a small peak-to-peak lightcurve variation or a nearly pole-on geometric aspect. Also, given the size of Orcus and the relatively slow rotation rate, we can most probably expect a circular shape from the relaxation of a fluid body in hydrostatic equilibrium.

To date, Orcus is known to have a single relatively large companion, Vanth, in a close circular orbit (see details in Table 1). According to Brown et al. (2010), the satellite is on a nearly face-on orbit (with respect to the Sun). Again, Orcus seems to be a unique case in an intermediate regime: larger KBOs have relatively small satellites in circular orbits, while small KBOs have very large satellites (Noll et al. 2008). As described by Brown et al. (2010), two scenarios could be invoked to explain the creation of the Orcus binary system which we will summarize below. In any case, because the orbit is circular, tidal evolution was at work at some point of the system's history. First, a catastrophic collision could be at the origin of the formation of Vanth, as hypothesized for Pluto-Charon (Canup 2005). Because of the large inclination of Vanth's orbit, Kozai cycling (Perets \& Naoz 2009) could also have occurred. A second scenario would then be an initial capture of Vanth, followed by large oscillations of eccentricity an inclination (Kozai cycling), and when the pericenter drops to a low enough value, tidal evolution can finally lead the orbit to its current circular shape. As noted by
Brown et al. (2010), a future discovery of any Orcus coplanar satellite would rule out this possibility (as for the Pluto-Charon system). Relative color measurements by the same team indicate that Vanth is significantly redder than Orcus (another unique property among KBO binaries), a characteristic that is currently difficult to understand in the framework of a formation by a giant impact.

In this work, we review the existing spectroscopic investigations of Orcus and objects with similar spectral and physical properties and search for additional constraints on the presence of volatiles (and maybe their irradiation products) on an object that might have retained some of its original content. We first report additional $0.4-2.5 \mu \mathrm{m}$ photometry and spectroscopy obtained from the Mauna Kea large telescopes. We present Hapke radiative transfer modeling of the spectral data, the testing for the presence of water ice, methane and ammonia ices (and their irradiation products, ethane and ammonium resp.) at the surface and discuss the results. Finally, we use a new 3D thermal evolution model dedicated to the interiors of KBOs (Guilbert-Lepoutre et al. 2010) to describe Orcus' thermal history over the age of the Solar System and the observable consequences of this history on the current surface.

\section{Observations and data reduction}

\subsection{Visible data}

Visible spectra of Orcus were acquired in visitor mode on 2005 April 9, at the Keck-1 telescope with the LRIS-RB (Low Resolution Imaging Spectrometer, Oke et al. 1995) instrument mounted at the Cassegrain focus. Beam-splitters separate the light between two arms, red and blue. The field of view (FOV) of both arms is $\sim 7^{\prime} \times 6.8^{\prime}$. The blue side is equipped with a mosaic of two $2 \mathrm{~K} \times 4 \mathrm{~K}$ Marconi (E2V) CCDs with a plate scale of $0.135^{\prime \prime} /$ pixel. The red arm has a Tektronik $2048 \mathrm{~K} \times 2048 \mathrm{~K}$ detector with a scale of $0.211^{\prime \prime} /$ pixel. We used the gratings $400 / 3400$ on the blue side and 400/8500 on the red side with dichroic 560 . The resulting wavelength coverage is $0.37-0.83 \mu \mathrm{m}$ with a resolution of $\sim 350$. We used a long slit of one arcsec width that we maintained along the parallactic angle during all the observations to minimize the loss of flux due to the atmospheric refraction. Arc images were obtained from $\mathrm{Hg} / \mathrm{Ne} / \mathrm{Cd} / \mathrm{Zn}$ lamp frames on the blue side and $\mathrm{Ne} / \mathrm{Ar}$ lamp frames on the red side. To avoid additional known distortion effects due to the telescope flexure, we acquired spectral flat-field and arc frames for each science telescope position.

We corrected the spectral images for bias, flat-field and cosmic rays with the IRAF (Tody 1986) spectroscopy pack, which we also used to calibrate the wavelength. For each instrument arm, we extracted the spectra using IRAF/APEXTRACT APALL task and merged them into a single $1 \mathrm{D}$ spectrum. It was corrected by a solar analog (HD 126053) observed close in airmass and time and normalized to unity around $0.55 \mu \mathrm{m}$. The resulting $2700 \mathrm{~s}$ spectrum (which is a combination of three individual spectra, $900 \mathrm{~s}$ each) is presented in Fig. 1. We do not believe that the slight turn-off at $0.42 \mu \mathrm{m}$ is real. We inspected the three individual spectra and found that although the overall spectral slope remains consistent from spectrum to spectrum, small variations were observed near the blue end of the individual spectra, which may be caused by the poor CCD sensitivity in this wavelength region. Therefore, we concluded that the slight turn-down feature that appears at $0.42 \mu \mathrm{m}$ in the combined spectrum of Orcus is not real, but an artifact. 
Table 2. Comparison of the visible spectral slope $\mathrm{S}$ (in $\% / 100 \mathrm{~nm}$ ) as computed from linear regression using the same algorithm on published data and our work over the common spectral range $(0.51-0.76 \mu \mathrm{m})$.

\begin{tabular}{llcclc}
\hline \hline Data reference & UT date & $r(\mathrm{AU})^{a}$ & $\alpha\left(^{\circ}\right)^{b}$ & Instrument & Computed S $(\% / 100 \mathrm{~nm})$ \\
\hline This paper & $2005-04-09$ & 47.67 & 0.99 & Keck + LRIS-RB & $2.01 \pm 0.54$ \\
Fornasier et al. (2009) & $2008-02-04$ & 47.81 & 0.48 & VLT + FORS2 & $1.75 \pm 0.57$ \\
de Bergh et al. (2005) & $2004-04-11$ & 47.62 & 1.02 & VLT + FORS2 & $1.42 \pm 0.74$ \\
Fornasier et al. (2004) & $2004-02-29$ & 47.61 & 0.46 & TNG + DOLORES & $1.80 \pm 0.67$ \\
\hline
\end{tabular}

Notes. ${ }^{(a)}$ Heliocentric distance from JPL Horizons ephemeris. ${ }^{(b)}$ Phase angle $\left(^{\circ}\right)$ from JPL Horizons ephemeris.

Table 3. Near-infrared photometry from Gemini+NIRI (this work) and near-infrared published colors from Orcus.

\begin{tabular}{lllllll}
\hline \hline UT date & $J \pm \sigma$ & $H \pm \sigma$ & $K \mathrm{~s} \pm \sigma$ & $J-H \pm \sigma$ & $H-K \pm \sigma$ & Reference \\
\hline 2005-02-20 & $17.86 \pm 0.08$ & $17.73 \pm 0.05$ & $17.82 \pm 0.05$ & $0.14 \pm 0.10$ & $-0.09 \pm 0.07$ & This paper \\
2005-02-21 & $17.82 \pm 0.04$ & $17.70 \pm 0.04$ & $17.81 \pm 0.04$ & $0.12 \pm 0.07$ & $-0.11 \pm 0.06$ & This paper \\
2005-02-22 & $17.85 \pm 0.05$ & $17.74 \pm 0.05$ & $17.77 \pm 0.06$ & $0.11 \pm 0.07$ & $-0.03 \pm 0.08$ & This paper \\
Average & $17.84 \pm 0.11$ & $17.72 \pm 0.09$ & $17.80 \pm 0.08$ & $0.12 \pm 0.14$ & $-0.08 \pm 0.12$ & This paper \\
Aver. 2004-04-11 to 21 & - & - & - & $0.13 \pm 0.05$ & $0.04 \pm 0.050$ & de Bergh et al. (2005) \\
\hline
\end{tabular}

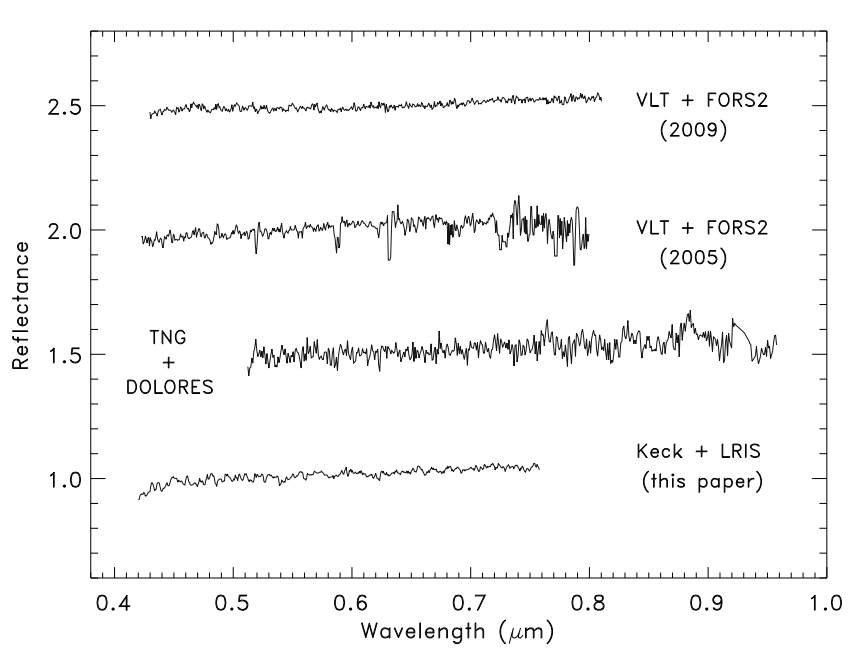

Fig. 1. A compilation of available visible spectra of Orcus, presented at a resolution of $R \sim 600$. From bottom to top: this paper, Fornasier et al. (2004), de Bergh et al. (2005), Fornasier et al. (2009). The three top spectra were shifted by 0.5 in reflectance for clarity.

We also acquired a series of $\mathrm{V}$ band images (through the broadband filter centered at $5437 \AA$ and with a $F W H M$ of $922 \AA$ ) to monitor the photometry over the course of the spectroscopy observations. Data reduction, photometric calibration and measurements were applied following a method described in Delsanti et al. (2001). We found an average value of $V=$ $19.06 \pm 0.03$, which corresponds to $m_{V}(1,1)=V-5 \log (r \Delta)=$ $2.32 \pm 0.03$ (with $r$ and $\Delta$ the helio- and geocentric distance respectively in AU). Rabinowitz et al. (2007) found a value of $H_{V}=2.33 \pm 0.03$ from their solar phase curve study. They published a phase correction coefficient for Orcus of $0.114 \pm$ $0.030 \mathrm{mag} \mathrm{deg}^{-1}$ in $V$. As we observed with a phase angle of $0.99^{\circ}$, our phase-corrected absolute magnitude is therefore $2.21 \pm 0.04$.

\subsection{Near-infrared data}

Data were acquired in queue mode at the Gemini North Telescope with the NIRI (Near InfraRed Imager) instrument on UT 2005 February 20-22 and UT March 1, (see Table 4). The detector is a $1024 \times 1024$ ALADDIN InSb array. We used the $\mathrm{f} / 6$ camera, which gives a FOV of $120^{\prime} \times 120^{\prime}$ and a pixel scale of 0.117 arcsec/pixel in imaging. Photometry was acquired through the broadband filters $J, H, K$ short, centered at 1.25 , $1.65,2.15 \mu \mathrm{m}$ respectively. We used the GEMINI NIRI package to produce the master dark and flat-field frames. To correct for the sky background contribution and recombine the sub-frames into the final image, we used the IRAF/XDIMSUM package. We measured the object flux using an aperture correction method (see Delsanti et al. 2004, for details on the complete procedure). Photometric coefficients (zero-point, extinction) were obtained from a least-square fit for the standard star flux measurements. Results are presented in Table 3. Our near-infrared colors are compatible with those of de Bergh et al. (2005).

We acquired the spectra through the 6-pixel wide slit and the f/6 J grism (1.00-1.36 $\mu$ m useful range with the "blue" slit, resolution of $\sim 480), \mathrm{H}$ grism $(1.43-1.96 \mu \mathrm{m}, R \sim 520)$, and $\mathrm{K}$ grism (1.90-2.49 $\mu \mathrm{m}, R \sim 520)$. Circumstances are detailed in Table 4. We used the usual AB nodding technique to acquire the sub-frames of a cube of data (the telescope was dithered by $\sim 20^{\prime \prime}$ in between sub-images). Solar analogs were observed to correct science spectra from the telluric lines and the contribution from the Sun's spectrum. We used the GEMINI GNIRS package to produce the ancillary frames (dark, flatfield, arc). Cubes of dithered spectra were corrected from the dark current, flatfield, and the distortion effects. The $\mathrm{AB}$ pairs were subtracted ( $\mathrm{A}-\mathrm{B}$ and $\mathrm{B}-\mathrm{A})$ to correct from the sky contribution and were re-aligned into a final composite $2 \mathrm{D}$ image. The $1 \mathrm{D}$ spectrum was extracted with the IRAF/APEXTRACT APALL task. Wavelength calibration was performed with IRAF/ONEDSPEC package.

\section{Results}

\subsection{A featureless neutral visible spectrum}

Orcus shows a linear and featureless spectrum (within the noise level) over the visible range, which is compatible with previously published data. Figure 1 shows the spectra available in the literature, presented at a resolution of $R \sim 600$ (data from this work and Fornasier et al. 2004, were degraded to match the $R \sim 600$ resolution of the FORS2 data). We computed a linear regression using the same algorithm for all published spectra and our work (on the original, non-degraded data), over the wavelength range that is common to all spectra $(0.51-0.76 \mu \mathrm{m})$ in order to compare the results: values of the spectral slope in 
Table 4. List of near-infrared Gemini+NIRI spectroscopy observations.

\begin{tabular}{llllll}
\hline \hline G. $^{a}$ & UT start date & Exp. $^{b}$ & Airmass & Solar An. & Airm. \\
\hline$J$ & $2005-02-2009: 43$ & 1200 & $1.10-1.12$ & HIP51125 & 1.24 \\
$H$ & $2005-03-0110: 54$ & 2160 & $1.22-1.41$ & HIP47071 & 1.24 \\
$K$ & $2005-02-2209: 38$ & 3600 & $1.09-1.13$ & HIP51125 & 1.28 \\
$K$ & $2005-02-2210: 52$ & 2100 & $1.15-1.23$ & HIP51125 & 1.28 \\
\hline
\end{tabular}

Notes. ${ }^{(a)}$ Grism. $J: 1.00-1.36 \mu \mathrm{m}, H: 1.43-1.96 \mu \mathrm{m}, K: 1.90-2.49 \mu \mathrm{m}$. (b) Exposure time in seconds.

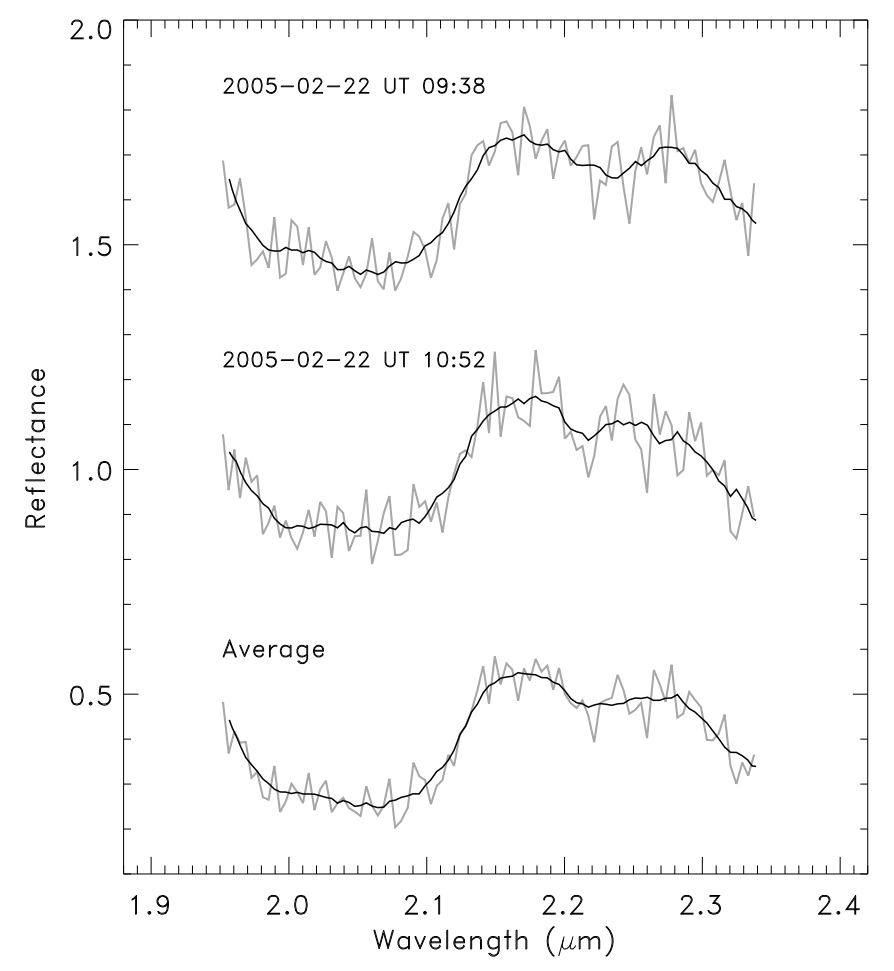

Fig. 2. Orcus spectra taken with the $\mathrm{K}$ grism on 2005-02-22. Top and bottom spectra were shifted by +0.6 and -0.6 resp. in reflectance for clarity. The solid line is a running mean to guide the eye (smooth box with a width of $\sim 55 \AA$ ).

$\% / 100 \mathrm{~nm}$ are reported in Table 2. They are compatible at one sigma level and are characteristic of a quasi-neutral surface with solar colors. We do not believe the slight turn-off at 0.4 micron is real as explained in Sect. 2.1. Orcus shows very consistent visible spectral properties over time, which strongly points to a homogeneous surface, at least in the optical.

\subsection{Crystalline water ice, ammonia, and methane?}

We adjusted the visible and near-infrared parts of the spectrum in reflectance using the $V$ magnitude computed in Sect. 2.1 and near-infrared photometry from 2005 February 20, (see Table 3 i.e. photometry that was observed simultaneously with the $\mathrm{J}$ band spectrum). In the $K$ band, we obtained two consecutive spectra on 2005 February 22, which are plotted at the resolution of the instrument in Fig. 2 along with the average spectrum. The complete reconstructed spectrum at the instrument resolutions from 0.40 to $2.35 \mu \mathrm{m}$ is presented in Fig. 4 (using the average of $K$-band spectral data).

In the near-infrared, the spectrum of Orcus shows deep absorption bands centered at $1.5 \mu \mathrm{m}, 1.65 \mu \mathrm{m}, 2.05 \mu \mathrm{m}$ (attributed
Table 5. Water ice near-infrared absorption band depths and central positions for Orcus.

\begin{tabular}{lll}
\hline \hline Data & Central $\lambda(\mu \mathrm{m})$ & Depth $(\%)$ \\
\hline H grism & 1.50 & $25.5 \pm 3.6$ \\
H grism & 1.65 & $13.2 \pm 4.5$ \\
K grism UT 09:38 & 2.05 & $28.2 \pm 3.6$ \\
K grism UT 10:52 & 2.05 & $22.2 \pm 6.7$ \\
\hline
\end{tabular}

to the presence of crystalline water ice) and smaller features around $2.2 \mu \mathrm{m}$. Measured water ice band depths and positions (using a continuum removal plus Gaussian fitting method) are reported in Table 5. Figure 3 shows a close-up of the data in the 1.4-2.4 $\mu \mathrm{m}$ range, the synthetic reflectance spectrum of different pure and hydrated ices, and some of their irradiation products (with $10 \mu \mathrm{m}$ size grains). From these, we can unambiguously identify crystalline water ice (with the broad $1.5 \mu \mathrm{m}$ and $2.0 \mu \mathrm{m}$ features and the $1.65 \mu \mathrm{m}$ absorption band). Methane or ammonia ices may account for the $2.2 \mu \mathrm{m}$ absorption band as surmised also by Barucci et al. (2008). However, hydrated and pure ammonia ices do not share the same features around $2.2 \mu \mathrm{m}$. The Orcus spectral shape beyond $2.23 \mu \mathrm{m}$ may also suggest the presence of methane ice. Ethane ice is also surmised, as it is an irradiation product from methane (Baratta et al. 2003): both components best described the near-infrared spectral behavior of Quaoar (Schaller \& Brown 2007a), which is very similar to that of Orcus. We also show a spectrum of ammonium $\left(\mathrm{NH}_{4}^{+}\right)$, as it is an irradiation product of ammonia and helped to reproduce the $2.2 \mu \mathrm{m}$ features in the spectrum of Pluto's satellite Charon (Cook et al. 2009).

\section{Radiative transfer modeling of the surface composition}

\subsection{Algorithm and parameters}

To better investigate the surface properties of Orcus, we ran a radiative transfer model following the theory of Hapke (1981, 1993) over the complete wavelength range available. This algorithm fits a synthetic reflectance spectrum to the data, using reflectance properties (optical constants) from a user-defined input list of chemical components, their relative amounts, and a grain size. When applying this method, one has to keep in mind that the best-fit synthetic spectrum obtained is not a unique solution to describe the object's surface composition and that some parameter values might lead to the best fit, although with no physical meaning. A detailed description of this model and its limits are presented in Merlin et al. (2010b).

We chose a symmetry parameter of $v=-0.4$ and a backscattering parameter $B=0.5$. These values are close to those computed by Verbiscer \& Helfenstein (1998) to describe the surfaces of the icy satellites of the giant planets. Finally, we computed the geometric albedo at zero phase angle from Eq. (44) of Hapke (1981). We used the Marqvardt-Levenberg algorithm to obtain the lower reduced $\chi^{2}$ value for the fit of the synthetic spectra to our data. Each synthetic spectrum is obtained using an intimate mixture of different compounds (from a user-defined input list). The free parameters are the grain size and the relative amount of each chemical compound. We emphasize that the results presented here are not unique and only show a possible surface composition. 


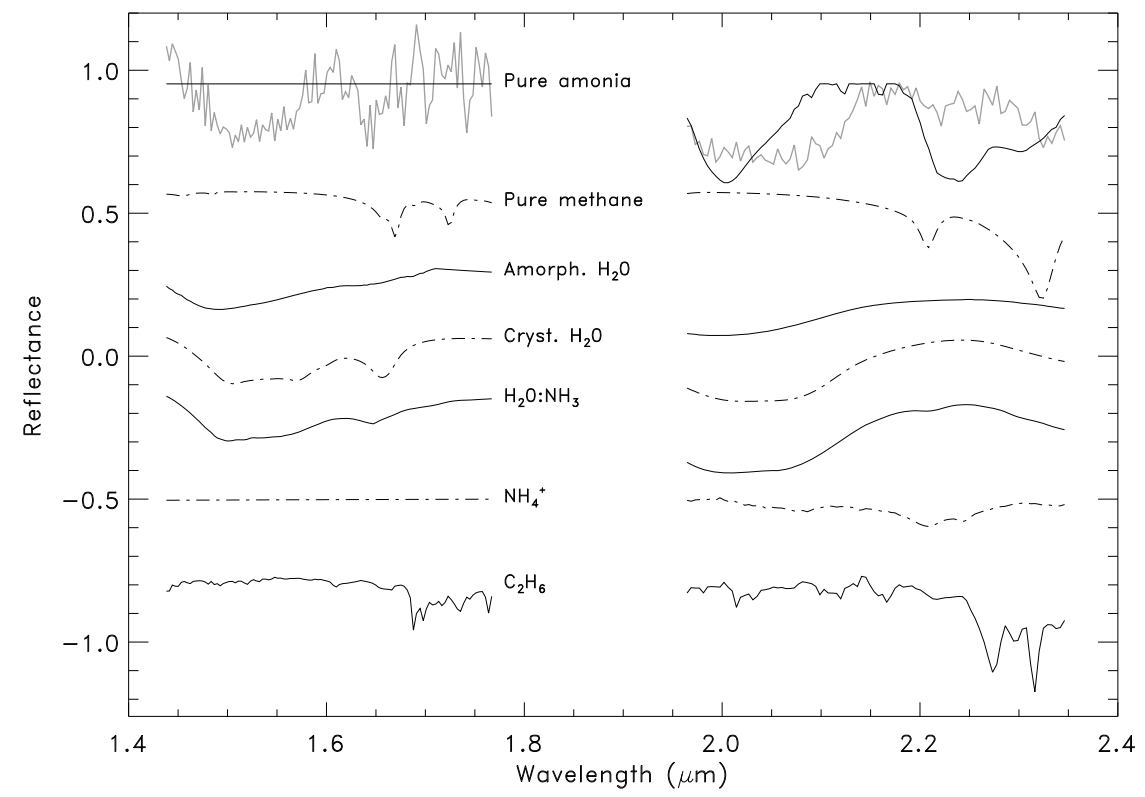

Fig. 3. A close up of Orcus spectrum in $H$ and $K$ bands (top, grey line) and the reflectance of, from top to bottom: pure ammonia and, shifted by several units for clarity, pure methane ice, amorphous water ice, crystalline water ice, hydrated ammonia $\left(\mathrm{H}_{2} \mathrm{O}: \mathrm{NH}_{3}, 99: 1\right)$, ammonium and ethane. Synthetic ice spectra were obtained using Hapke theory (Hapke 1981, 1993) with the optical constants quoted in this paper and a particle size of $10 \mu \mathrm{m}$.

\subsection{The choice of the chemical components}

As input to the radiative transfer model, we used optical constants of several ices at low temperature (close to $40 \mathrm{~K}$ ) that are likely to be present on the surface of an icy body located at $\sim 50 \mathrm{AU}$ such as:

- water ice (Grundy \& Schmitt 1998);

- pure methane ice (Quirico \& Schmitt 1997);

- pure ammonia ice (Schmitt et al. 1998);

- diluted ammonia ice (Ted Roush, personal comm.);

- pure methanol ice (Quirico \& Schmitt, personal comm.).

We also used optical constants of a dark compound such as amorphous black carbon (Zubko et al. 1996), which is able to reproduce the low albedo of the majority of TNOs (Stansberry et al. 2008). Space weathering is known to be responsible for the alteration of the KBOs surfaces with time through the action of galactic cosmic rays ions, solar wind plasma ions and energetic particles, (Strazzulla et al. 1991; Cooper et al. 2003). The effect on ices is documented from laboratory experiments (for instance Brunetto et al. 2006): the fresh and bright icy mantle becomes spectrally redder and darker with irradiation. Without any better constraints on the nature of the irradiation products present on the surface of these objects, we decided to use tholins, which reproduce the spectral slope behavior best in the visible (in our case, Titan tholins were found to be the more appropriate; this compound is generated from the irradiation of mixtures of $\mathrm{N}_{2}$ and $\mathrm{CH}_{4}$, Khare et al. 1984).

Additional spectroscopic observations of Orcus in the near infrared ( $H+K$ band only, from Barucci et al. 2008) showed deep absorption features at 1.5 and $2.0 \mu \mathrm{m}$ and shallow bands at $1.65 \mu \mathrm{m}$ and close to $2.2 \mu \mathrm{m}$. The presence of crystalline water ice is evident in the $1.65 \mu \mathrm{m}$ feature. The weak $2.2 \mu \mathrm{m}$ feature is much more difficult to interpret (owing to the detection level and multiple candidate components). These authors tested Hapke models of ammonia hydrate or pure methane ices (separately): none of these attempts could satisfactorily reproduce the shape of the $2.2 \mu \mathrm{m}$ feature, although ammonia hydrate was the favored explanation. In this work, we will try to better reproduce the general shape of our spectrum on the full 0.4-2.4 $\mu \mathrm{m}$ range with the help of the Hapke modeling of mixtures of ices listed above, assuming an albedo of 0.2 (Stansberry et al. 2008). Below, we will not discuss in detail the various relative quantities listed in Table 6 , because we consider Hapke model results as only qualitatively indicative of the possible candidate components. The reasons are that: (i) synthetic models, even when accurately fitted to the data, do not provide a unique, definitive solution; (ii) the SNR of the data currently available for Orcus do not allow this detailed interpretation; (iii) the optical constants from materials used here where measured in conditions close to those that should prevail on Orcus, but some of them are missing and approximations were used.

\subsection{A simple water ice mixture}

Given the obvious features of water ice in its crystalline state in our data, our first attempt was to use a simple mixture of amorphous and crystalline water ice at $\sim 40 \mathrm{~K}$. Again, Titan tholins were used only to reproduce the spectral shape in the optical range (as in all models of this work): this compound is certainly not present as such at the surface of Orcus. Similarly, amorphous black carbon was only used as a mean to match the low measured albedo. The corresponding best-fit model is plotted in the bottom of Fig. 4, the quantities are tabulated in Table 6 (model 1).

The large path lengths of amorphous water ice should be taken with great care. Indeed, when alternatively using a blue component to fit the continuum (using for instance the reflectance properties of kaolinite from the ASTER database, http: //speclib.jpl.nasa.gov/search-1/mineral), the path lengths and the proportions of amorphous water ice in the final model drastically drop. This indicates that crystalline water ice is most probably dominating the mixture. All previous work on Orcus reports the presence of water ice (Fornasier et al. 2004; de Bergh et al. 2005; Trujillo et al. 2005), and the most recent higher SNR data show the presence of crystalline water ice (Barucci et al. 2008, and this work). The crystalline water ice fraction for this model is comparable to the one reported in Barucci et al. (2008) (about 15\%). The depth of the absorption feature at $1.65 \mu \mathrm{m}$ depends on the crystalline water ice 


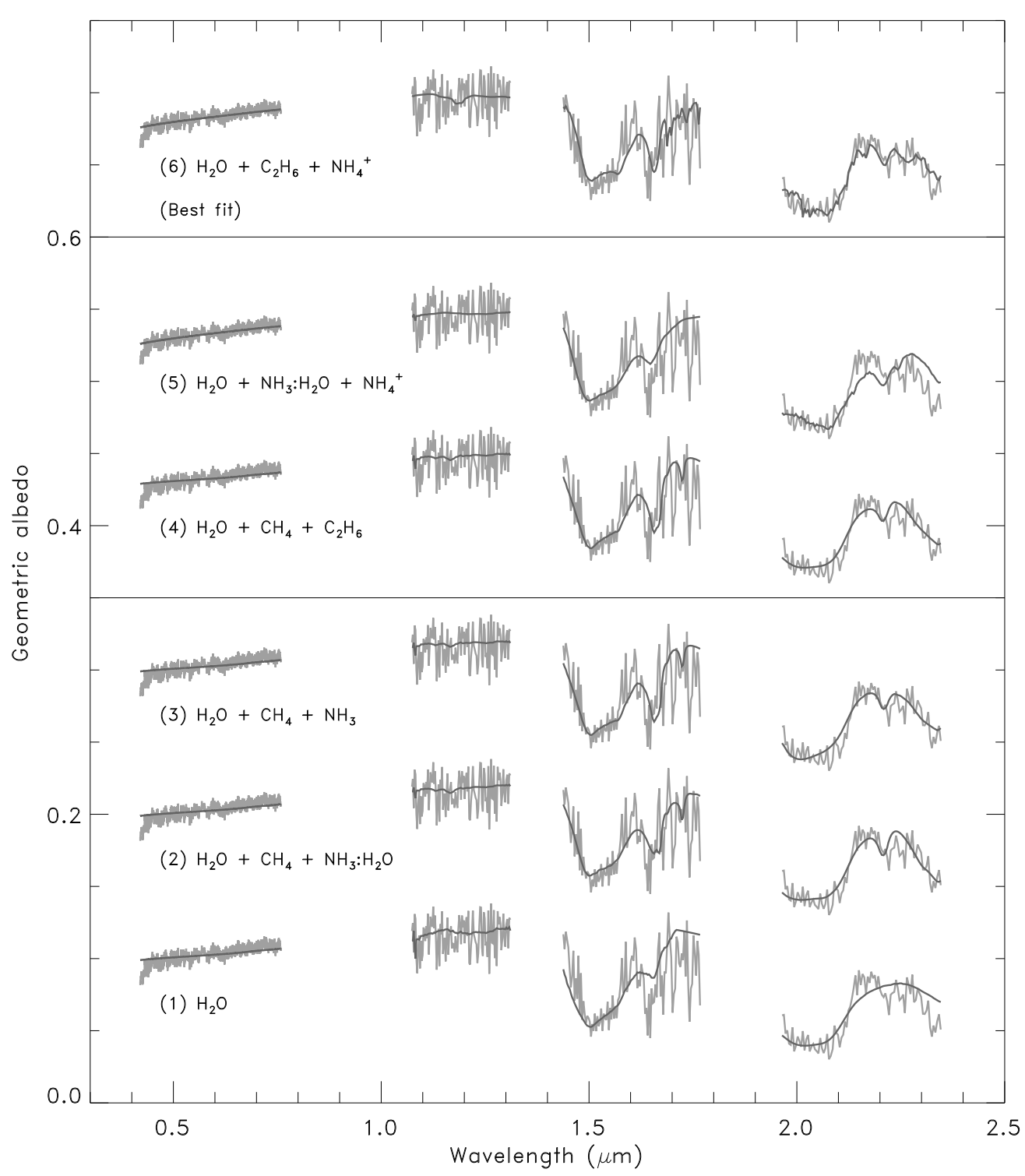

Fig. 4. Visible and near-infrared spectrum of Orcus (grey line) with synthetic spectra superimposed (solid black line). Spectra are shifted in albedo for clarity from -0.1 to about +0.5 (except model 2). Quantitative description of the models and reduced $\chi^{2}$ are presented in Table 6 . The bottom spectrum shows that a water ice-only model (Haumea-like surface) fails at describing all of Orcus features, especially in the $K$ band, although water ice is the likely dominant component. The two models above are the best-fit results from our next investigation step involving volatiles: they include a mixture of methane and ammonia (pure of diluted) in addition to water ice (in its amorphous and crystalline state). The middle part of the plot illustrates the models including ammonia or methane and their respective irradiation products (ammonium and ethane). The top part shows our overall best-fit result: a mixture of water ice, ammonium $\left(\mathrm{NH}_{4}^{+}\right)$, and ethane $\left(\mathrm{C}_{2} \mathrm{H}_{6}\right)$.

Table 6. Quantitative description of the chemical composition models with the relative percentage of each component.

\begin{tabular}{llllllll}
\hline \hline Components & Model 1 & Model 2 & Model 3 & Model 4 & Model 5 & Model 6 & Reference \\
\hline Amorphous $\mathrm{H}_{2} \mathrm{O}$ & $13 \%(500)$ & $5 \%(600)$ & $7 \%(400)$ & $9 \%(370)$ & $5 \%(300)$ & $1 \%(200)$ & Grundy \& Schmitt (1998) \\
Crystalline $\mathrm{H}_{2} \mathrm{O}$ & $13 \%(40)$ & $3 \%(100)$ & $13 \%(160)$ & $12 \%(150)$ & $3 \%(50)$ & $19 \%(200)$ & Grundy \& Schmitt (1998) \\
${\mathrm{Pure} \mathrm{NH}_{3}}$ & - & - & $2 \%(120)$ & - & - & - & Schmitt et al. (1998) \\
$\mathrm{NH}_{3}: \mathrm{H}_{2} \mathrm{O}(1: 99)$ & - & $13 \%(150)$ & - & - & $11 \%(300)$ & - & T. Roush (Priv. comm) \\
$\mathrm{Pure}_{\mathrm{CH}}$ & - & $6 \%(800)$ & $5 \%(800)$ & $5 \%(800)$ & - & - & Quirico \& Schmitt (1997) \\
Amorphous C & $71 \%(10)$ & $71 \%(10)$ & $71 \%(10)$ & $71 \%(10)$ & $71 \%(10)$ & $58 \%(10)$ & Zubko et al. (1996) \\
Titan tholin & $3 \%(10)$ & $2 \%(10)$ & $2 \%(10)$ & $3 \%(10)$ & $0 \%(10)$ & $1 \%(10)$ & Khare et al. (1984) \\
$\mathrm{C}_{2} \mathrm{H}_{6}$ & - & - & - & $0 \%(20)$ & - & $7 \%(600)$ & Quirico \& Schmitt (1997) \\
$\mathrm{NH}_{4}^{+}$ & - & - & - & - & $9 \%(800)$ & $13 \%(800)$ & J. Cook (Priv. comm.) \\
Reduced $\chi^{2}$ & 1.78 & $\mathbf{1 . 2 9}$ & $\mathbf{1 . 3 2}$ & 1.35 & 1.64 & $\mathbf{1 . 2 3}$ & \\
\hline
\end{tabular}

Notes. Grain size in micron is indicated in parenthesis. Models are sorted on Fig. 4 from bottom (model 1) to top (model 6). 
abundance and temperature of the surface. At low temperature (close to that of Orcus, $\sim 40 \mathrm{~K}$ ), a deep absorption feature as observed in our spectrum (or in previous works) suggests a high crystalline-to-amorphous water ice ratio.

This first attempt shows that a simple mixture of water ice approximately reproduces the general spectral behavior of Orcus in the near-infrared, but fails to describe the more subtle features (at $1.65 \mu \mathrm{m}$ and around $2.2 \mu \mathrm{m}$ ). Orcus therefore has a water dominated surface, but most probably hosts additional absorbing substances, such as volatiles, in smaller amounts.

\subsection{Adding methane and ammonia ices}

For this second step, we tested various ices that could absorb around $2.2 \mu \mathrm{m}$, contribute to the blue slope long-ward $2.3 \mu \mathrm{m}$ and could possibly be present on a $\sim 40 \mathrm{~K} \mathrm{KBO}$ (see also Fig. 3 and Sect. 3.2). In particular, ammonia hydrate has an absorption feature around $2.21 \mu \mathrm{m}$ : it was detected on Uranus' satellite Miranda (Bauer et al. 2002) and possibly identified on Quaoar (Jewitt \& Luu 2004) and Orcus (previous work by Barucci et al. 2008). Methane (dominating the surface composition of large KBOs, such as Pluto, Eris or Makemake) is also an interesting candidate, with its absorption feature around $2.2 \mu \mathrm{m}$ and blue reflectance in the 2.3-2.4 $\mu \mathrm{m}$ range. We also included methanol in our tests (pure $\mathrm{CH}_{3} \mathrm{OH}$ from Quirico \& Schmitt, priv. comm.)

At this stage, best-fit results were obtained using a mixture of water ice (amorphous and crystalline), methane and ammonia (pure or diluted). The resulting models 2 and 3 are plotted in Fig. 4 (see also Table 6). They have an equivalent reduced $\chi^{2}$ and reproduce almost equally the general shape of the near infrared spectrum. The agreement of the synthetic spectra to our data is however not totally satisfactory around $1.65 \mu \mathrm{m}$ and $2.2 \mu \mathrm{m}$, although the slope beyond $2.3 \mu \mathrm{m}$ is now correctly mimicked. Only traces of methane and ammonia are sufficient to significantly improve the fit with respect to a simple water ice mixture. We tested the mixtures used by Barucci et al. (2008) against our data, i.e. trying separately methane, then ammonia hydrate (respectively mixed with water ice) but obtained a worse fit than models 2 and 3. In particular, the $2.2 \mu \mathrm{m}$ band was poorly reproduced in both cases, and for the ammonia hydrate model, the synthetic spectrum had a reflectance that was too high beyond $2.3 \mu \mathrm{m}$.

From a theoretical point of view, ammonia and methane could be major volatiles in the solar nebula at the distance where Orcus might have formed (Lewis 1972). Ammonia on one hand is known to lower the temperature of the melting point when mixed with water ice. Methane on the other hand is almost insoluble in aqueous liquids at low pressures (no electric dipole moment). At the central pressure of Orcus (few kilo-bars), its solubility should nonetheless reach about $1 \%$. Methane could therefore trigger so-called explosive aqueous cryovolcanism in the presence of ammonia and water (Kargel 1992, 1995). This process has been introduced to explain the cryovolcanism observed on Triton (Kargel \& Strom 1990). Indeed, the bubble content of liquid melts would increase as they propagate toward the surface through cracks: the solubility of methane in these melts depend on the pressure, which decreases as the melts reach the surface. If the bubble content becomes sufficient, the melts would eventually disintegrate into a gas-driven spray producing the explosive cryovolcanism.

\subsection{Search for daughter species of methane and ammonia}

Ethane is an irradiation product (from UV photolysis) of solid ethane (Baratta et al. 2003), and is expected to be involatile at $\sim 40 \mathrm{~K}$. Therefore, once produced by solar irradiation, it should remain at the surface of the object and still be observable. Moore $\&$ Hudson (2003) report that the production of $\mathrm{C}_{2} \mathrm{H}_{6}$ is more efficient when $\mathrm{CH}_{4}$ is pure. $\mathrm{C}_{2} \mathrm{H}_{6}$ was suggested at the surface of Quaoar (Schaller \& Brown 2007a) and detected on dwarf planet Makemake (Brown et al. 2007a). Since our data could be compatible with traces of methane at the surface of Orcus, we decided to further investigate the contribution of $\mathrm{C}_{2} \mathrm{H}_{6}$. We used the optical constants from Quirico \& Schmitt (1997). Following a similar reasoning, we decided to test the presence of ammonium $\left(\mathrm{NH}_{4}^{+}\right)$, which can be produced for instance by irradiation of pure $\mathrm{NH}_{3}$ or mixtures of water and ammonia as experienced by (Moore et al. 2007). Other species can be produced from the irradiation of $\mathrm{NH}_{3}$ or $\mathrm{NH}_{3}: \mathrm{H}_{2} \mathrm{O}$, such as hydroxylamine $\left(\mathrm{NH}_{2} \mathrm{OH}\right)$; unfortunately, the corresponding weak infrared features are not easily detectable. In this test, we used optical constants of $\mathrm{NH}_{4}^{+}$ derived by Cook (priv. comm.) from the studies by Moore et al.

We therefore tested ammonia and methane separately along with their respective irradiation products. Model 4 (middle quadrant of Fig. 4) shows a mixture of water ice, methane, and ethane, while model 5 includes water ice, ammonia hydrate, and ammonium. The methane option (model 4 ) gives a lower reduced $\chi 2$ and overall better fit than model 5 , and is visually and statistically equivalent to models 2 and 3 . However, ethane is finally found not to contribute to the final mixture $(0 \%)$ when mixed with only $\mathrm{CH}_{4}$ and water ice, which tells us that methane is itself an interesting component to describe the $H$ and $K$ band data. The ammonia and ammonium mixture model is not satisfactory to improve the overall fit of Orcus spectrum (with respect to other models), but the structure of the $2.2 \mu \mathrm{m}$ band is worth of interest.

\subsection{Best-fit results and conclusions}

For this last step we aimed at better describing the data overall, in particular in the $2.2 \mu \mathrm{m}$ region, using results (and possible candidates) derived from the previous investigation steps. Our best-fit result overall is displayed as model 6 (top of Fig. 4) and includes a mixture of water ice mostly in its crystalline state $(\sim 20 \%)$, ammonium $(\sim 13 \%)$ and traces of ethane $(\sim 7 \%)$. The overall shape of the Orcus spectrum is correctly reproduced, in particular in the $K$ band with both the $2.05 \mu \mathrm{m}$ large water ice band and the $2.2 \mu \mathrm{m}$ region more correctly fitted. The presence of ammonium also better reproduces the visible slope. The $1.65 \mu \mathrm{m}$ feature was never completely correctly reproduced, which could be related to the SNR of the data. Our conclusion is that the irradiation products $\mathrm{NH}_{4}^{+}$and $\mathrm{C}_{2} \mathrm{H}_{6}$ greatly helps to describe the data in the 1.4-2.4 $\mu \mathrm{m}$ region, although both parent species, as a mixture of ammonia (pure or diluted) and methane cannot be firmly excluded (model 2 and 3 of equivalent statistical level). We believe that the four species are probably present as traces at the surface of Orcus. Therefore, further spectroscopic studies of Orcus with significanlty higher SNR (especially in the $2.2 \mu \mathrm{m}$ region) would improve our knowledge about the presence of the various ices (such as methane and ammonia) and related chemical products and processes on the surface of this object.

\subsection{Surface composition variations over time?}

We saw that the neutral, featureless (within the noise level) visible spectral properties are consistent over a timespan of about 
A\&A 520, A40 (2010)

Table 7. Comparison of orbital and physical properties of some intermediate-large size water-bearing objects of interest.

\begin{tabular}{|c|c|c|c|c|c|}
\hline Parameter & Orcus & Charon & (208996) $2003 \mathrm{AZ}_{84}$ & Quaoar & Haumea \\
\hline Dynamical Class $^{a}$ & $3: 2$ & $3: 2$ & $3: 2$ & Classical & Classical \\
\hline Orbit inclination $\left({ }^{\circ}\right)^{b}$ & 20.6 & 17.1 & 13.6 & 8.0 & 28.2 \\
\hline Semi-major axis $(\mathrm{AU})^{c}$ & 39.2 & 39.6 & 39.4 & 43.5 & 43.0 \\
\hline$q(\mathrm{AU})^{d}$ & 30.2 & 29.7 & 32.3 & 41.7 & 34.5 \\
\hline$Q(\mathrm{AU})^{e}$ & 48.1 & 49.6 & 46.5 & 45.2 & 51.5 \\
\hline$r(\mathrm{AU})^{f}$ & 47 & 31 & 45 & 43 & 51 \\
\hline Diameter $(\mathrm{km})$ & $\begin{array}{l}946 \pm 70^{(1)} \\
940 \pm 70^{(3)}\end{array}$ & $1207 \pm 3^{(2)}$ & $686 \pm 95^{(1)}$ & $\begin{array}{l}850 \pm 200^{(1)} \\
910 \pm 100^{(6)} \\
1260 \pm 190^{(4)}\end{array}$ & $\begin{array}{l}1150 \pm 200^{(1)} \\
\sim 2000 \times 1500 \times 1000^{(5)}\end{array}$ \\
\hline$p_{V}(\%)^{g}$ & $\begin{array}{l}19.7 \pm 3^{(1)} \\
28 \pm 4^{(3)}\end{array}$ & $\sim 40^{(7)}$ & $12.3 \pm 4^{(1)}$ & $\begin{array}{l}19.9 \pm 10^{(1)} \\
17.2 \pm 5^{(6)} \\
9.2 \pm 4^{(4)}\end{array}$ & $\begin{array}{l}80 \pm 15^{(1)} \\
60-80^{(8)}\end{array}$ \\
\hline Vis. slope $(\% / 100 \mathrm{~nm})^{h}$ & $\sim 2$ & - & $\sim 3$ & $\sim 28$ & $\sim 0$ \\
\hline Dominant ice at surface & $\mathrm{H}_{2} \mathrm{O}$ (cryst) & $\mathrm{H}_{2} \mathrm{O}$ (cryst) & $\mathrm{H}_{2} \mathrm{O}$ (cryst) & $\mathrm{H}_{2} \mathrm{O}$ (cryst) & $\mathrm{H}_{2} \mathrm{O}$ (cryst) \\
\hline Volatiles and other & $\begin{array}{l}\mathrm{NH}_{4}^{+}+\mathrm{C}_{2} \mathrm{H}_{6} \\
\mathrm{NH}_{3}: \mathrm{H}_{2} \mathrm{O}+\mathrm{CH}_{4} \\
\mathrm{NH}_{3}+\mathrm{CH}_{4} \\
\end{array}$ & $\begin{array}{l}\mathrm{NH}_{4}^{+}+\mathrm{CH}_{4}+\mathrm{NH}_{3}: \mathrm{H}_{2} \mathrm{O}^{(9)} \\
\mathrm{NH}_{3}: \mathrm{H}_{2} \mathrm{O}, \mathrm{NH}_{3}(13) \\
\mathrm{NH}_{4} \mathrm{OH}^{(13)}\end{array}$ & $\mathrm{CH}_{3} \mathrm{OH} ?^{(10)}$ & $\begin{array}{l}\mathrm{CH}_{4}+\mathrm{C}_{2} \mathrm{H}_{6}^{(11)} \\
\mathrm{CH}_{4}+\mathrm{C}_{2} \mathrm{H}_{6}+\mathrm{N}_{2}^{(12)}\end{array}$ & - \\
\hline
\end{tabular}

Notes. ${ }^{(a)}$ Using classification by Gladman et al. (2008). ${ }^{(b)}$ With respect to ecliptic plane, from the Minor Planet Center. ${ }^{(c)}$ From the Minor Planet Center. ${ }^{(d)}$ Perihelion from the Minor Planet Center. ${ }^{(e)}$ Aphelion from the Minor Planet Center. ${ }^{(f)}$ Approximate current heliocentric distance. ${ }^{(g)}$ Visible geometric albedo. ${ }^{(h)}$ Slope of the visible spectrum from Fornasier et al. (2009) and references therein, except for Orcus: this paper.

References. (1) Stansberry et al. (2008), (2) Sicardy et al. (2006) and references therein, (3) Brown et al. (2010), (4) Brown \& Trujillo (2004), (5) Brown (2008), (6) Brucker et al. (2009), (7) computed from Buie et al. (2010), (8) Rabinowitz et al. (2006), (9) Cook et al. (2009), (10) Guilbert et al. (2009), (11) Schaller \& Brown (2007a), (12) Dalle Ore et al. (2009), (13) Merlin et al. (2010a) and references therein.

4 years. It is almost impossible to phase the different spectra with the rotational ligthcurves available because they were not observed close enough in time. However, as Orcus does not show dramatic lightcurve effects (see Sect. 1) and is expected to be a spherical object, it is reasonable to think that the surface properties are rather homogeneous over the surface in the optical. In the near-infrared, according to the most recent, higher SNR data (Barucci et al. 2008, and this work), crystalline water ice was always present (and in the same roughly proportions as in the indicative Hapke modeling). A $2.2 \mu \mathrm{m}$ feature was also systematically shown to be there in the most recent works, with slightly different structures, although we believe they are roughly consistent within the noise. Until significantly higher SNR data are available and show more subtle changes (as for instance Charon), we can conclude that Orcus' spectral properties are rather homogeneous over its surface in the near-infrared at the currently available resolution.

\subsection{Contribution of the satellite}

The known properties of the Orcus binary system are summarized in Table 1. Because of the small separation of the pair (0.26" Noll et al. 2008), it is impossible to separate the contribution of both components in our ground-based observations. The color measurements performed with the Hubble Space Telescope (from which the binary system can be resolved) by Brown et al. (2010) show that Vanth displays significantly redder visible and near-infrared colors than Orcus. The contribution of the satellite to the total flux is $8 \%$ in the visible and a bit more in the near-infrared. Thus, we do not expect large variations in the water ice content of the main body. Ammonia, methane, and their irradiation products, if confirmed, should be present at the surface of the primary object only, as Vanth is probably too small to have retained volatile ices at its surface. Given that the satellite's near-infrared color index is redder than that of Orcus (as measured by Brown et al. 2010), we can expect that the satellite will have a lower water ice content, if any.

\section{Comparison with other water-bearing KBOs of interest}

In this section we look at the surface expression of water (more specifically in its crystalline form) and additional traces of volatiles on some other large-intermediate water bearing objects. We try to point out common properties and differences, and in the case of Quaoar, infer general clues about its possible history and interior. The compared properties are summarized in Table 7.

\section{1. (50000) Quaoar}

The KBOs (90482) Orcus and (50000) Quaoar seem to share the same type of near-infrared spectrum: dominated by crystalline water ice, with small amounts of volatiles (Schaller \& Brown 2007a). Besides, they are both intermediate-size KBOs with similar albedo (Stansberry et al. 2008): their study could thus shed light on the possible transition between large methanedominated objects (like Pluto, Eris, Sedna and Makemake) and smaller water-ice-dominated ones. Nonetheless, these two objects might be very different when it comes to their thermal and dynamical history. Moreover, their visible spectral properties differ: Quaoar, with its $\sim 28 \% / 100 \mathrm{~nm}$ slope, is part of the objects hosting ultra-red material (>25\%/100 nm, Jewitt 2002) on its surface. Tables 7 and 8 show a comparison between various physical and dynamical properties of Orcus and Quaoar.

In this table, the maximum surface temperature is computed from a thermal balance for the whole surface including different energy inputs such as: the incoming solar energy,

$E_{\odot}=(1-\mathcal{A}) \frac{C_{\odot}}{d_{H}^{2}} \cos (\xi)$, 
Table 8. Comparison of Orcus and Quaoar additional parameters.

\begin{tabular}{|c|c|c|c|}
\hline & Parameter & Orcus & Quaoar \\
\hline \multirow{4}{*}{ : } & Dynamical class & Plutino & Classical KBO \\
\hline & Semi-major axis $^{1}(\mathrm{AU})$ & 39.163 & 43.467 \\
\hline & Eccentricity $^{1}$ & 0.227 & 0.040 \\
\hline & $P(\mathrm{yr})$ & 245.5 & 286.9 \\
\hline \multirow{3}{*}{$\begin{array}{l}\dot{0} \\
\dot{0} \\
\dot{a} \\
\dot{\vec{\lambda}} \\
\dot{0}\end{array}$} & Density $\left(\mathrm{g} \mathrm{cm}^{-1}\right)$ & $\begin{array}{l}1.9 \pm 0.4^{a} \\
1.5 \pm 0.3^{b}\end{array}$ & $>2.8^{c}$ \\
\hline & $T_{\text {surf }} \max .(\mathrm{K})$ & $40-50$ & $42-46$ \\
\hline & Insulation per orb. $(\mathrm{J})$ & $\sim 2.0 \times 10^{21}$ & $\begin{array}{l}1.5 \times 10^{21} \\
3.3 \times 10^{21}\end{array}$ \\
\hline$\dot{\tilde{E}}$ & $\begin{array}{l}\text { Brightness } \\
\text { fraction }(\%)\end{array}$ & $8^{a}$ & $0.6^{a}$ \\
\hline
\end{tabular}

References. 1: values from the Minor Planet Center. a: Brown (2008); b: Brown et al. (2010); c: Fraser \& Brown (2009).

(with $\mathcal{A}$ the Bond albedo, $C_{\odot}$ the solar constant, $d_{H}$ the heliocentric distance and $\xi$ the local zenith angle), the thermal emission $\varepsilon \sigma T^{4}$ (with $\varepsilon$ the thermal emissivity, $\sigma$ the Stefan-Boltzmann constant and $T$ the temperature), the radial heat flux and lateral heat fluxes, considering a thermal conductivity of about $10^{-2} \mathrm{~W} \mathrm{~m}^{-1} \mathrm{~K}^{-1}$. This thermal balance is computed for about 50 orbits with a full $3 \mathrm{D}$ thermal evolution model to get the resulting temperatures (see Appendix B of Guilbert-Lepoutre et al. 2010). The insulation per orbit (in J), is computed with the formula (Prialnik et al. 2004)

$Q=\frac{\pi L_{\odot}}{2 \sqrt{G M_{\odot}}} \frac{R^{2}}{\sqrt{a\left(1-e^{2}\right)}}$,

with $L_{\odot}$ and $M_{\odot}$ the solar luminosity and mass respectively, $G$ the gravitational constant and $R$ the object's radius. Depending on the radius value we use for Quaoar, two different values are obtained for $Q$. The values for Orcus and Quaoar (and surface temperature) are consistent, although their spectral response to solar illumination is very different. Indeed, despite the similar visual albedo and near infrared spectral properties, Orcus' visible spectrum is neutral, whereas Quaoar's spectrum has a red slope: they most probably have different thermal properties, in terms of their spectral response to solar illumination, light absorption and subsequent heating.

Quaoar is a classical KBO and as such should have been formed in situ, or was moderately pushed outward during Neptune's migration (Gomes 2003). Therefore, it should have been formed on a larger timescale than Orcus, implying potentially less accretional heating. Orcus is a Plutino and might have been formed closer to the Sun than its current location (Malhotra 1993, 1995). We can thus suspect that its accretion was faster and involved more heat. The early thermal history of both objects in this sense might be quite different. Nonetheless, because of their rather large sizes and densities, they could have both reached temperatures sufficient to melt water ice and produce a differentiated internal structure (i.e. after heating by short-lived radiogenic elements, the interior should be stratified, with a differentiated core and layers enriched/impoverished in volatile ices closer from the surface).

The abundance of radiogenic elements is directly related to the dust mass fraction within the body: the denser an icy body is, the more heat will be released upon the decay of these radiogenic elements. Considering Quaoar's high density, we can assess that it might have suffered from severe thermal modifications due to the decay of long-lived radiogenic elements such as ${ }^{40} \mathrm{~K},{ }^{232} \mathrm{Th}$, ${ }^{235} \mathrm{U},{ }^{238} \mathrm{U}$. Therefore, the methane observed at its surface could have been placed there in a quite recent past, although the object should be geologically dead for millions years. The subsequent surface evolution should have been dominated by spaceweathering processes. Orcus is less dense than Quaoar: a thermal evolution model is applied to test if long-lived radiogenic elements could have heated the object in the last million years, so that the effects on the surface would still be observable today (see Sect. 6).

\subsection{The Haumea collisional family}

(136108) Haumea is a $2000 \mathrm{~km}$-class fast-rotating elongated dwarf planet from the classical Kuiper belt with the highest water ice surface content currently known among KBOs and a neutral visible spectrum (see Pinilla-Alonso et al. 2009; Merlin et al. 2007, and references therein). Brown et al. (2007b) identified several objects with similar spectral properties in a dynamical area that is close to Haumea. Because Haumea's unusual rotational properties are compatible with a giant impact, it was soon suggested that these objects are members of the first collisional family identified in the Kuiper belt. Repeated spectral and photometric studies (Snodgrass et al. 2009; Barkume et al. 2008; Rabinowitz et al. 2008; Pinilla-Alonso et al. 2008; Schaller \& Brown 2007b; Pinilla-Alonso et al. 2007) aimed at refining the family properties and members list.

From these studies, we see that despite the presence of water ice and the neutral visible spectrum, the other properties of the Haumea collisional family differ from those of Orcus. Haumea itself has a much higher albedo (0.6-0.84 Rabinowitz et al. 2006; Stansberry et al. 2008), and density (2600-3340 $\mathrm{kg} \mathrm{m}^{-3}$ Rabinowitz et al. 2006) and much deeper water ice absorption bands. Pinilla-Alonso et al. (2009) showed that it has a spectrally homogeneous surface best described by a simple intimate mixture of amorphous and crystalline water ice and that it is probably depleted of any other ices. As a consequence, cryovolcanism cannot be invoked as possible a re-surfacing process, whereas it might play a role in Orcus' evolution. In this work, we also showed that a simple water ice mixture (Haumea-type surface) fails to completely reproduce Orcus' spectral behavior in the near-infrared (see model 1).

\subsection{Charon}

Pluto's larger satellite Charon is a $\sim 1200 \mathrm{~km}$ size body with a water ice dominated surface. Charon and Orcus are of similar size and density (1.5-1.9 $\left.\mathrm{g} \mathrm{cm}^{-1}\right)$, on a similar orbit, and share common spectral properties. However, their albedos are quite different, as Charon was measured to have a visible geometric albedo of $\sim 0.40$ (computed from Buie et al. 2010). Crystalline water ice is unambiguously detected and leads to a surface temperature estimate of $40-50 \mathrm{~K}$ (Cook et al. 2007), which is in the same range as that of Orcus ( $44 \mathrm{~K}$ from Barucci et al. 2008). Another component absorbing at $2.21 \mu \mathrm{m}$ is preferably attributed to ammonia-type ices from detailed Hapke modeling (Merlin et al. 2010a, and references therein), and it seems that the surface is variegated (differences between the Pluto and antiPluto hemispheres). Recent high SNR $K$-band spectral studies (Cook et al. 2009) suggested for the first time that ammonium could be present at the surface of Charon, as it helps to better model the absorption features they detected around 2.21 and $2.24 \mu \mathrm{m}$. Their best-fit Hapke model includes a mixture of water, 
ammonium, methane, and ammonia hydrate. In this work, we tried to fit to our data a similar synthetic spectrum: we obtained a reduced $\chi^{2}$ of 1.68 (e.g. of lower quality than our best-fit options), although the general spectral features of Orcus were correctly reproduced. This confirms the similarities of Orcus and Charon bulk surface properties. Also, Desch et al. (2009) identified from their 1D-spherical thermal model of Charon that cryovolcanism is the most probable mechanism to provide fresh ices on the surface. From our own thermal model (see Sect. 6), we draw a similar conclusion for the case of Orcus.

\subsection{Plutino (208996) $2003 A Z_{84}$}

(208996) $2003 \mathrm{AZ}_{84}$ is, like Orcus, a binary Plutino wandering in a 30-50 AU heliocentric region. It is presently located at a similar heliocentric distance, $\sim 46 \mathrm{AU}$ (wrt $\sim 48 \mathrm{AU}$ for Orcus). (208996) has a quasi-neutral featureless spectrum in the optical, with a spectral slope of $1.6 \pm 0.6 \% / 100 \mathrm{~nm}$ (Fornasier et al. 2009, and references therein). Guilbert et al. (2009) confirmed the presence of water ice (Barkume et al. 2008) in its crystalline phase from near-infrared spectroscopy, and noted a possible absorption feature around $2.3 \mu \mathrm{m}$. The SNR of the data is not sufficient to identify the corresponding absorbing substance. However, the general spectral behavior of Plutino (208996) is close to that of Orcus, and joint studies deserve to be conducted in the future.

\section{A thermal-evolution model of Orcus}

\subsection{The model}

We used a thermal-evolution model in order to evaluate the possibility for Orcus to undergo some internal activity events such as cryovolcanism in a recent past, so that the effects might still be observable at the surface. From input orbit, size, albedo, density, porosity, formation delay and type of radiogenic elements, we simulated the evolution of temperature over the age of the Solar System for the whole object. In this model, the heat diffusion equation (Eq. (3)) is solved using a fully 3D scheme, considering internal heating due to the crystallization of amorphous water ice, as well as heating due to the decay of several shortand long-lived radiogenic elements. We therefore need to solve the equation

$\rho_{\text {bulk }} c \frac{\partial T}{\partial t}+\operatorname{div}(-\kappa \overrightarrow{\operatorname{grad}} T)=\mathcal{S}$,

where $T$ is the temperature distribution we need to determine, $\rho_{\text {bulk }}$ the object's bulk density, $c$ the material heat capacity, $\kappa$ its effective thermal conductivity and $S=Q_{\text {rad }}+Q_{\text {cryst }}$ the two heat sources. The radiogenic heat source is described by

$Q_{\mathrm{rad}}=\sum_{\mathrm{rad}} \varrho_{d} X_{\mathrm{rad}} H_{\mathrm{rad}} \frac{1}{\tau_{\mathrm{rad}}} \exp \left(\frac{-t}{\tau_{\mathrm{rad}}}\right)$,

with $\varrho_{d}$ the dust bulk density, $X_{\text {rad }}$ the initial mass fraction of a particular radiogenic element in the dust, $H_{\text {rad }}$ the heat released by this radiogenic element per unit mass upon decay, $\tau_{\text {rad }}$ its decay time, and $t$ the time. We consider different short- and long-lived radiogenic elements, presented in Table 9, using data adapted from Castillo-Rogez et al. (2007, and references therein) for ordinary chondrites.

The heat source due to the crystallization of amorphous water ice is described by

$Q_{\text {cryst }}=\lambda(T) \varrho_{a} H_{\mathrm{ac}}$,
Table 9. Physical parameters of various short-period (SP) and longperiod (LP) radiogenic elements considered in the model.

\begin{tabular}{ccccc}
\hline \hline Element & $X_{\text {rad }}(t=0)^{a}$ & $\begin{array}{c}\tau_{\text {rad }}{ }^{b} \\
\text { (years) }\end{array}$ & $\begin{array}{c}H_{\text {rad }}{ }^{c} \\
\mathrm{~J} \mathrm{~kg}^{-1}\end{array}$ \\
\hline \multirow{3}{*}{$\mathrm{SP}$} & ${ }^{26} \mathrm{Al}$ & $6.7 \times 10^{-7}$ & $1.05 \times 10^{6}$ & $4.84 \times 10^{12}$ \\
& ${ }^{60} \mathrm{Fe}$ & $2.5 \times 10^{-7 /-8}$ & $2.16 \times 10^{6}$ & $5.04 \times 10^{12}$ \\
& ${ }^{53} \mathrm{Mn}$ & $2.8 \times 10^{-8}$ & $5.34 \times 10^{6}$ & $4.55 \times 10^{12}$ \\
\hline & ${ }^{40} \mathrm{~K}$ & $1.2 \times 10^{-6}$ & $1.80 \times 10^{9}$ & $1.66 \times 10^{12}$ \\
$\mathrm{LP}$ & ${ }^{232} \mathrm{Th}$ & $6.0 \times 10^{-8}$ & $2.02 \times 10^{10}$ & $1.68 \times 10^{13}$ \\
& ${ }^{235} \mathrm{U}$ & $9.0 \times 10^{-9}$ & $1.02 \times 10^{9}$ & $1.82 \times 10^{13}$ \\
& ${ }^{238} \mathrm{U}$ & $2.9 \times 10^{-8}$ & $6.45 \times 10^{9}$ & $1.92 \times 10^{13}$ \\
\hline
\end{tabular}

Notes. Values adapted from Castillo-Rogez et al. (2007). ${ }^{(a)}$ Initial mass fraction of the radiogenic element. ${ }^{(b)}$ Decay time. ${ }^{(c)}$ Heat released by this radiogenic element per unit mass upon decay.

with $\varrho_{a}$ the amorphous water ice bulk density. This phase transition releases a latent heat $H_{\mathrm{ac}}$, whose value is $9 \times 10^{4} \mathrm{~J} \mathrm{~kg}^{-1}$ (Klinger 1981), with a rate measured by Schmitt et al. (1989) of:

$\lambda(T)=1.05 \times 10^{13} \mathrm{e}^{-5370 / T} \mathrm{~s}^{-1}$.

The heat diffusion equation is then expanded on the real spherical harmonics basis. The resulting equation is numerically solved using an implicit stable Crank Nicolson scheme. A full description of this model can be found in Guilbert-Lepoutre et al. (2010).

Several physical parameters have to be initialized before simulating the thermal evolution of Orcus. We first assume that the object is on its current orbit for the whole simulation and is placed there right after its formation time. This does not reflect the true dynamical evolution of Orcus, but might not strongly affect the resulting general thermal behavior (as detailed in the next paragraphs). Orcus is then assumed to have a $475 \mathrm{~km}$ radius, a $19.9 \%$ albedo (Stansberry et al. 2008), and a bulk density of $1900 \mathrm{~kg} \mathrm{~m}^{-3}$ (Brown 2008). By assuming a residual porosity of $10 \%$ (Durham et al. 2005), this density leads to a mixture made of $77 \%$ of dust and $23 \%$ of water ice (mass fractions), the dust being homogeneously distributed within the ice matrix. The resulting thermal properties are: the thermal conductivity $\kappa=0.18 \mathrm{Wm}^{-1} \mathrm{~K}^{-1}$, the heat capacity $c=10^{3} \mathrm{Jkg}^{-1} \mathrm{~K}^{-1}$.

Since heating due to the accumulation of gravitational potential energy is usually not important, even for large KBOs such as Pluto (McKinnon et al. 1997), we neglect this heat source in this model. We also assume that the objects undergo a cold accretion: the accretional heating is thus neglected, although it could be a very important heat source in the case of Orcus.

\subsection{Results}

\subsubsection{Effect of short-lived radiogenic elements}

The formation time of KBOs is of critical importance when it comes to the study of their differentiation due to heating by short-lived radiogenic elements. These elements indeed decay during the accretion period, thus providing much less potential heat when the bodies are formed (and our simulations start). There is still a wide range of available values for this formation time, depending on models. For example, Weidenschilling (2004) suggested that $50 \mathrm{~km}$-radius objects could be formed in less than one million years inside $30 \mathrm{AU}$. Recent simulations performed by Kenyon et al. (2008) show that 1000 km-radius 
bodies could be formed in $5-10 \times 10^{6} \mathrm{yr}$ within the $20-25 \mathrm{AU}$ region.

With the aim of constraining Orcus' state of differentiation (primordial and during its lifetime), we applied the model considering various formation delays as input. The initial temperature is $30 \mathrm{~K}$ (cold accretion), and the radiogenic elements initial mass fraction is calculated using the following formula, to account for their decay during accretion

$X_{\text {rad }}\left(t_{F}\right)=X_{\text {rad }}(0) \mathrm{e}^{-t_{F} / \tau_{\text {rad }}}$,

with $t_{F}$ the considered formation delay, and $X_{\text {rad }}(0)$ found in Table 9. All SP and LP elements listed there are taken as input, and we distinguish the case of the high and low ${ }^{60} \mathrm{Fe}$ mass fraction. Our simulations over the age of the Solar System $\left(4.5 \times 10^{9} \mathrm{yr}\right)$ show that Orcus could have a physically differentiated structure -including a melted core, crystalline water ice mantle, and a pristine surface layer composed of amorphous ice and dust- if formed in less than about $5 \times 10^{6} \mathrm{yr}$. The melting point of water is indeed achieved within the body in the first millions years of the simulations. If we assume that ammonia is also present in the mixture, then this formation time limit is slightly increased to about $7 \times 10^{6}$ years, since the presence of ammonia depresses the melting point from a few $\mathrm{K}$ to almost $100 \mathrm{~K}$ depending on its abundance (Kargel 1992, and references therein).

Figure 5 shows the temperature evolution inside Orcus at various depths (center, $1 \mathrm{~km}$-deep, $500 \mathrm{~m}$-deep) considering the highest mass fraction of short-lived ${ }^{60} \mathrm{Fe}$ and a formation delay of $1 \mathrm{Myr}$. Although this formation delay might seem too short for such a large object, the corresponding results show the greatest temperature variations and the steepest thermal gradients expected, to put an upper limit to all thermal processes. The radiogenic heating is homogeneous inside the body, but the surface is in thermal equilibrium with the external medium. This results in the propagation of a cold wave from the surface toward deeper layers. As a consequence, the curves corresponding to the $1 \mathrm{~km}-$ depth and 500 m-depth show a maximum temperature after a few millions years of the simulations: the body then starts to cool slowly.

Another consequence is that a layer of amorphous water ice is maintained at the surface of the object: the 1 m-deep layer temperature reaches the water crystallization threshold at some point, while the $500 \mathrm{~m}$-deep layer does not complete the crystallization process. The propagation of the cold wave from the surface to the interior is illustrated by Fig. 6, which shows the radial temperature profile across the body for different simulated times. The thickness of this pristine material layer depends on the formation delay that we consider: the shorter the delay, the thinner the layer. We find that the thickness could be as low as several hundreds of meters ( $\sim 500 \mathrm{~m}$ for the 1 Myr delay) or as thick as a few tens of kilometers $(\sim 10 \mathrm{~km}$ for the 10 Myr delay). Most of the volume is therefore made of crystalline water ice. Our simulations also show that the threshold for the solid-liquid phase transition of water is reached within most of the volume (if formed fast enough): the high temperatures needed to keep liquid water inside Orcus are moreover maintained for long periods such as $2 \times 10^{9}$ years.

\subsubsection{Effect of long-lived radiogenic elements}

What would happen if Orcus was formed in more than 10 Myr? In this case, the effect of short-lived radiogenic elements would be nonexistent. However, radiogenic heating due to the decay of
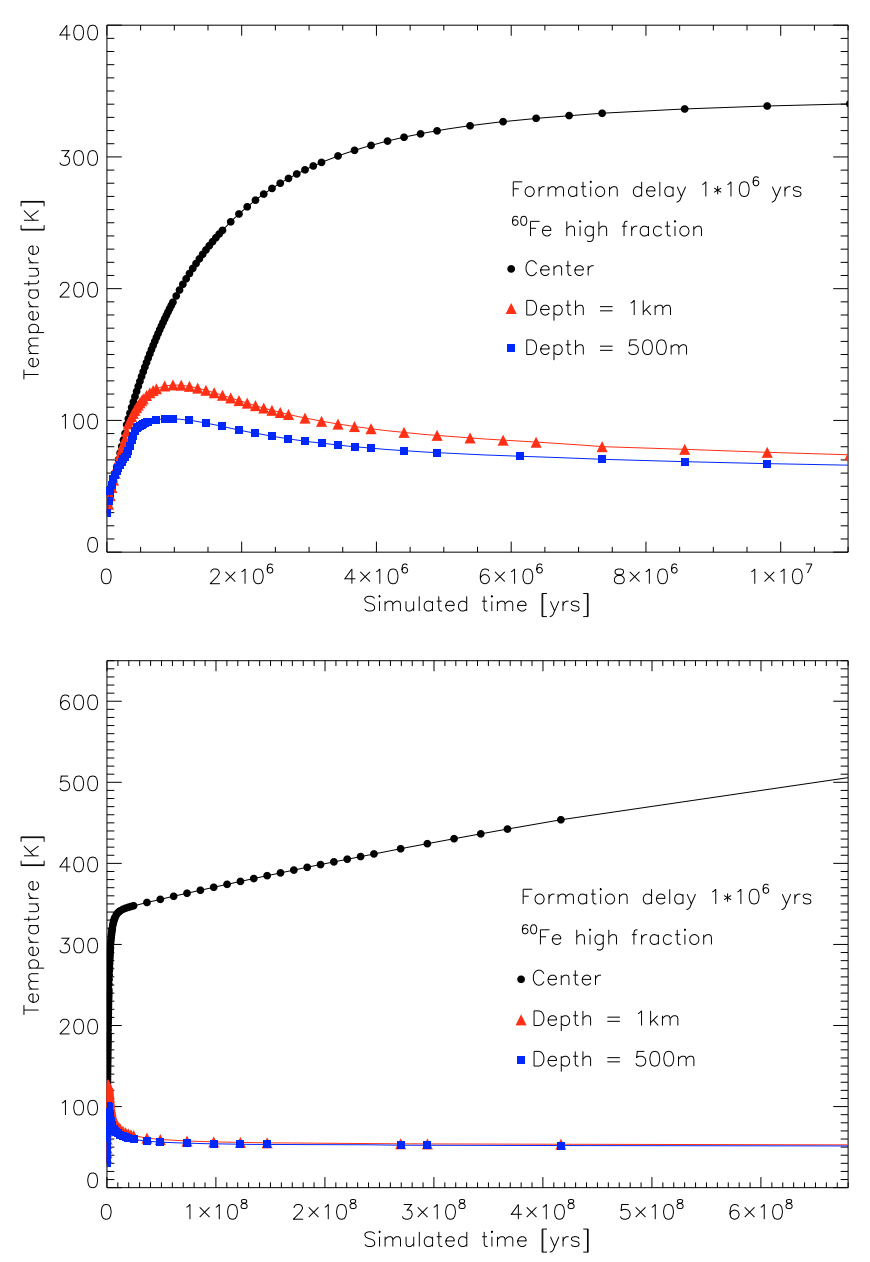

Fig. 5. Evolution of Orcus' central temperature (black circles) as a function of the simulated time in the case of a formation delay of 1 million years and a high mass fraction of radiogenic element ${ }^{60} \mathrm{Fe}$. Triangles and square curves correspond to the temperature at a depth of $1 \mathrm{~km}$ and $500 \mathrm{~m}$ resp. Top graph zooms on the first $\sim 10 \mathrm{Myr}$ of simulation.

${ }^{40} \mathrm{~K},{ }^{232} \mathrm{Th},{ }^{235} \mathrm{U}$, and ${ }^{238} \mathrm{U}$ would still be inevitable. This would even be of the greatest importance for Orcus, whose thermal timescale,

$\tau_{\text {th }}=\frac{R^{2} \rho_{\text {bulk }} c}{\pi^{2} \kappa}$

exceeds the age of the Solar System (assuming the previous thermal physical parameters). Nonetheless, the value of the thermal conductivity is critical for this calculation, and a variation of one magnitude could reduce the cooling time to less than $10^{9}$ years.

We therefore applied our model including only the long-lived radiogenic elements ${ }^{40} \mathrm{~K},{ }^{232} \mathrm{Th},{ }^{235} \mathrm{U}$, and ${ }^{238} \mathrm{U}$. The evolution of the body's central temperature is shown on Fig. 7, compared to the evolution under the effect of both long- and short-lived elements. Even without the high heating power of short-lived elements, the internal temperature increases, reaching the melting point of water ice within the first billion years. Please keep in mind that our mixture contains a high fraction of dust that might not reflect the true composition of Orcus. In this case Orcus suffers from considerable heating, while it could actually be much more moderate. Our simulations show that in the case of an important heating by long-lived radiogenic elements, the melting point of water ice could be reached within about $60 \%$ of the body, while the melting point of an ammonia-water mixture 


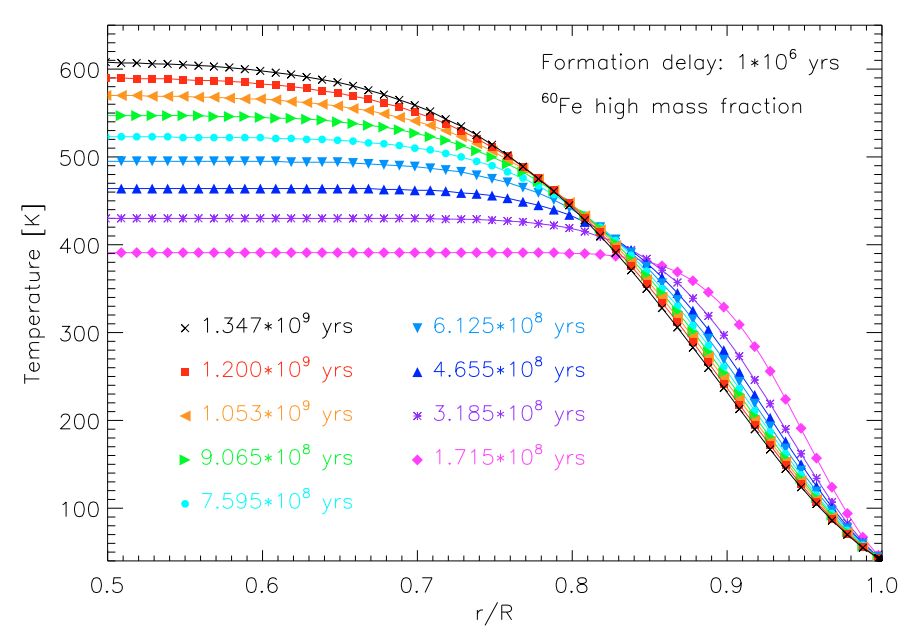

Fig. 6. Temperature $(\mathrm{K})$ as a function of the object's depth for various evolution times. This plot illustrates how the outside medium cools the surface layers deeper with time (for SP and LP radiogenic elements, high ${ }^{60} \mathrm{Fe}$ mass fraction.)

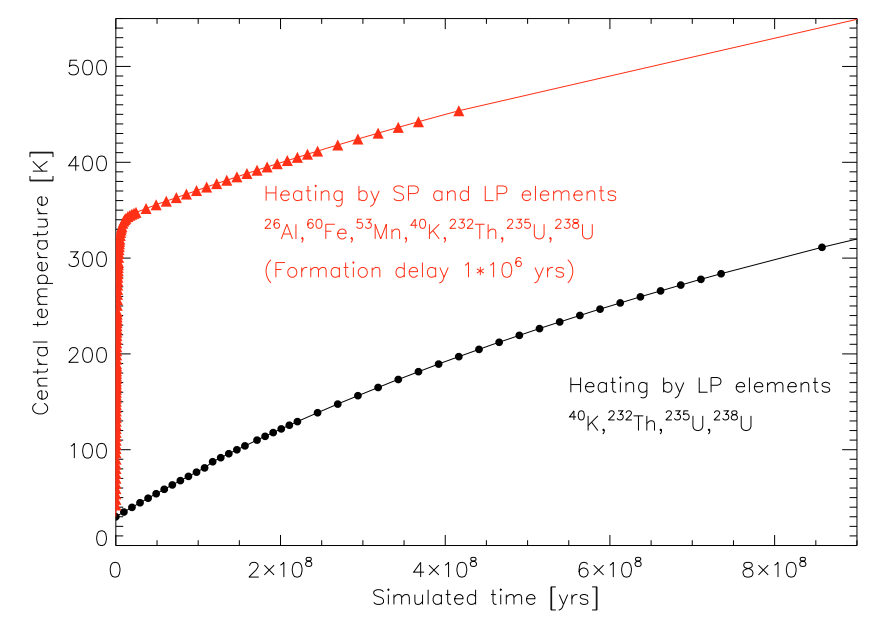

Fig. 7. Central temperature $(\mathrm{K})$ as a function of the simulated time, showing the different contributions from $\mathrm{SP}+\mathrm{LP}$ radiogenic elements, and LP only.

would be reached within $80 \%$ of the body. This is illustrated in Fig. 8, which shows radial temperature profiles for different simulated times. As in the cases considered in the previous section, a layer of amorphous water ice is maintained at the surface of Orcus. Its thickness is about $5-6 \mathrm{~km}$.

\subsection{Implications}

\subsubsection{Limitations}

Our model has several limitations that need to be kept in mind when interpreting the results. The main issue is that the liquid phase or differentiation is not accounted for: the model is not suitable anymore when the temperature threshold for solid-liquid phase transition is reached. The results we provide should therefore be regarded as qualitative evolutionary trends rather than a quantitative description of Orcus' thermal evolution. Accounting for sources of substantial cooling such as convection movements would probably induce moderate or even low temperature at the present time. Moreover, we considered

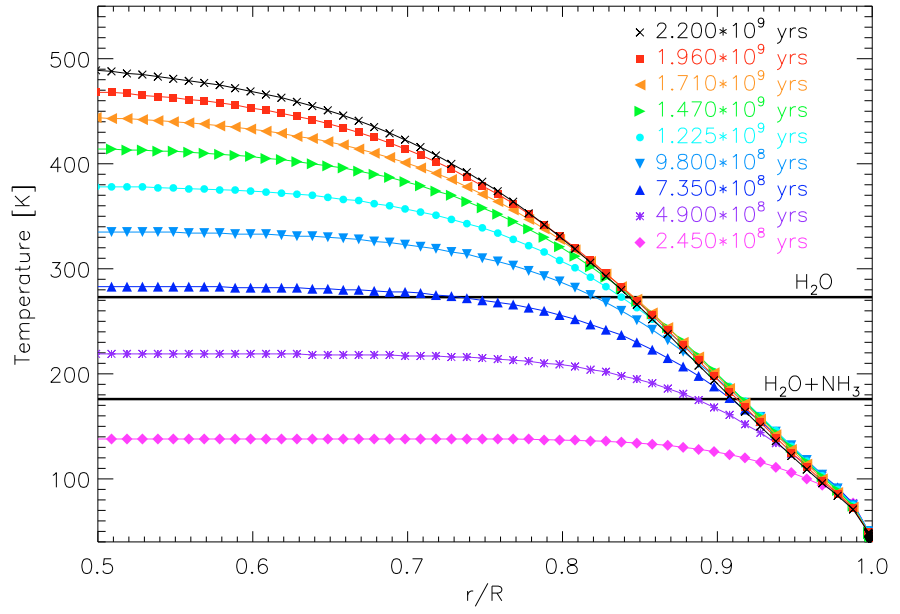

Fig. 8. Temperature $(\mathrm{K})$ as a function of the object's depth for various evolution times (the lower limit is the case of radiogenic heat source from long-lived elements only). Horizontal lines show the temperature above which $\mathrm{H}_{2} \mathrm{O}$ or $\mathrm{H}_{2} \mathrm{O}+\mathrm{NH}_{3}$ are in the liquid phase.

a water ice and dust mixture which contains a high fraction of dust, which in turn induces a high heating power by radiogenic elements. This dust fraction might actually be lower, and some thermal properties such as the thermal conductivity might also be reduced by a large factor in reality. However, even when considering low heating, our simulations show that Orcus should have experienced a cryovolcanism episode in its life, especially if ammonia is present in its bulk composition.

We did not take into account the possible contribution from the satellite in our simulations, as it is currently not possible to constrain it. However, if the binary system was created by a giant impact, we can expect that a large fraction of the main body's surface layers was removed, thus exposing interior material. Furthermore, the heat from the impact should contribute to a temporal increasing of Orcus' upper layers temperature. Tidal heating was not accounted for either, and might play a role in Orcus thermal evolution if the satellite is found to be large enough (current rough estimates from Brown et al. 2010, give a size of the satellite of $1 / 3$ to half the primary).

\subsubsection{A past cryovolcanic event}

Under the effect of an efficient heating due to radiogenic elements, a liquid phase (water+ammonia, if ammonia is present) is produced, and could have reached layers close to the surface, eventually freezing, and/or even propagating through cracks within the ice matrix, thus erupting at the surface (Stevenson 1982). This process could be even more efficient with methane, which can trigger explosive cryovolcanism (Kargel 1992, 1995). Our spectral data show a compatibility with traces of ammonia and methane at the surface of Orcus, which means that the interior of the object most probably contains ammonia. There is nonetheless no easy way to infer the internal abundance of ammonia from the surface composition, since the latter would not reflect the internal composition, due to the chemical modifications induced by space weathering processes. As described by Desch et al. (2009), the presence of only a few percent of ammonia in the interior (by weight, relative to water) can efficiently lower the viscosity of the ammonia-water mixture once is has melted. Please note however that large amounts of ammonia might be incompatible with a high dust/ice ratio such as the 
one used on our model (Wong et al. 2008). Our simulations show that we cannot rule out a cryovolcanic episode in Orcus' past life, which probably supplied most of the observed crystalline water ice of the surface. Surface erosion with impact cratering could also contribute to bring to the surface some fresh material, including crystalline water ice, ammonia, and methane, refrozen in subsurface layers, but to a much lesser extent (as computed by Cook et al. 2007, for Charon).

\subsubsection{Origin and surviving of crystalline water ice}

The initial state of water ice in the Solar System is still a matter of debate:

- if water ice was initially crystalline when Orcus formed, then it could have stayed so over the age of the Solar System in the object's interior. Space weathering processes would have induced its progressive amorphization at the surface (partial, as we will see below, or complete);

- if water ice was initially amorphous when Orcus formed (as we assumed), then our simulations show that it would have been rapidly crystallized compared to the age of the Solar System for most of the objects' volume. A layer of pristine amorphous ice (a few kilometers thick in the canonical case) would have been maintained at the surface. In this case, the crystalline water ice would only appear at the surface if a supply mechanism was at work at some point of the history, or if the erosion by impacts removed the amorphous ice over few kilometers (which is currently believed to be an inefficient process to explain the detectable quantities of crystalline water ice on Orcus, other KBOs and giant planets' satellites, as computed by Cook et al. 2007, for Charon). However, a more detailed modeling of the impact environment of Orcus needs to be conducted before completely ruling out the resurfacing effects of impacts.

In our particular case, the detection of large amounts of crystalline water ice at the surface of Orcus could indicate that heating from radiogenic decay and the crystallization of amorphous ice had been an efficient process, thus bringing the crystallization front close to the surface, as shown by our thermal evolution model. But most importantly, our results show that Orcus' thermal history is compatible with a past cryovolcanic event, which is an efficient supply mechanism of fresh ices to the surface. We can therefore envision that most of the crystalline water ice we currently observe are the actual leftovers from such a geological event. Indeed, recent laboratory experiments by Zheng et al. (2009), show that the $1.65 \mu \mathrm{m}$ crystalline water ice absorption band is able to survive ion irradiation doses up to $160 \pm 30 \mathrm{eV}$ for $T \geq 30 \mathrm{~K}$. This phenomenon is temperature-dependent, and becomes even more efficient at higher temperatures. This means that the amorphization of the crystalline ice is not complete, and starting at $50 \mathrm{~K}$, there is a balance dominated by thermal recrystallization. From the irradiation doses determined by Cooper et al. (2003) for the optical thickness of $0.1 \mathrm{~mm}$ (i.e. the layers probed by near infrared spectroscopy), Zheng et al. computed for a $\mathrm{KBO}$, that the $1.65 \mu \mathrm{m}$ band should be able to survive during about 6 billion yrs, e.g. longer than the Solar System age. If this result is confirmed, it means that the 40-50 K surface of Orcus still holds traces of a past cryovolcanic event in the form of detectable crystalline water ice, and that additional resurfacing processes are not necessarily needed.

\section{Summary}

We provided new $0.4-2.35 \mu \mathrm{m}$ reflectance spectra and nearinfrared photometry of Plutino (90482) Orcus. Our visible spectrum is featureless (within the SNR of the data) with a neutral slope $(\sim 2 \% / 100 \mathrm{~nm})$. These results are consistent with previously published works and point (within the noise level) to a homogeneous surface at least in the optical. Our near-infrared spectra confirm the presence of water ice in its crystalline state (thanks to the $1.65 \mu \mathrm{m}$ absorption band) at the surface of Orcus, as well as the presence of a $2.2 \mu \mathrm{m}$ feature, which cannot be readily identified owing to both the detection level and the multiple candidates. This band might show a double structure, which deserves to be further investigated from significantly higher SNR studies than currently available. These general spectral properties are shared with Pluto's satellite Charon and Plutino (208996) $2003 \mathrm{AZ}_{84}$.

Radiative transfer Hapke models show that a simple mixture of water ice (amorphous and crystalline) is not sufficient to properly describe the data in the $H$ and $K$ band. Orcus therefore does not have a Haumea-type surface, although $\mathrm{H}_{2} \mathrm{O}$ should be the dominant ice. Additional absorbents should be invoked, which may be volatiles and their possible irradiation products. Further Hapke modeling shows that the $2.2 \mu \mathrm{m}$ region and overall spectrum from 0.4 to $2.35 \mu \mathrm{m}$ are best described by an intimate mixture of ammonium $\left(\mathrm{NH}_{4}^{+}\right)$and traces of ethane $\left(\mathrm{C}_{2} \mathrm{H}_{6}\right)$, which are most probably solar irradiation products, in addition to water ice (mostly in its crystalline state). The presence of ammonium even helps to better reproduce the visible spectrum, an effect which was not expected a priori. We find also that our data are compatible with Hapke models of an equivalent (slightly lower) statistical level including a mixture of water ice, methane, and ammonia (pure of diluted); the latter are the parent species of ethane and ammonium. Methane is particularly interesting to reproduce the blue slope longward $\sim 2.3 \mu \mathrm{m}$, which might also be the shortward wing of an additional absorption feature. Focused studies should be conducted in close connection with laboratory investigations to better understand the methane and ammonia chemistry.

In this work, we showed that the Orcus spectrum could be compatible with the presence of volatile ices on its surface (as also hypothesized by Barucci et al. 2008), and their involatile irradiation products (first invoked here). Our findings merits confirmation by higher SNR data, and we believe that Plutino (208996) $2003 \mathrm{AZ}_{84}$ and Orcus deserve joint detailed studies, to be also connected with Charon properties, as they share common spectral properties. Schaller \& Brown (2007b) developed a simple model of atmospheric escape to predict how a given $\mathrm{KBO}$ can preserve a primordial surface inventory of $\mathrm{CO}, \mathrm{N}_{2}$ and $\mathrm{CH}_{4}$. Orcus, Charon and Quaoar are located in a relative narrow region in their Fig. 1, showing the equivalent temperature $(\mathrm{K})$ as a function of the object's diameter $(\mathrm{km})$. Orcus is located in the "all volatiles lost" region, where methane and nitrogen ices in particular should have completely escaped from the surface. That our Orcus data could be compatible with the presence of volatiles (here $\mathrm{CH}_{4}$ ) would imply that (i) such an atmospheric model is too simple to describe the actual volatile content of a particular object (as it was emphasized by the authors in their conclusions); (ii) Orcus might be larger than the size we currently know from far-IR data (and Herschel Space Observatory observations might allow us to answer this question, although from the Schaller and Brown model, a factor of $\sim 1.7$ would be needed); (iii) there is a supply of volatile ices from the interior of the object; (iv) the actual situation is a combination of the previous points. 
We investigated the thermal properties of Orcus over its lifetime to understand how crystalline water ice can be produced and test if geological events such as cryovolcanism can be possible and provide fresh ices to the surface. We used a 3D thermal evolution model by Guilbert-Lepoutre et al. (2010) that simulates the temperature of the whole object as a function of its size, density, porosity, orbit, formation delay and radiogenic elements primordial inventory $\left({ }^{26} \mathrm{Al},{ }^{60} \mathrm{Fe},{ }^{53} \mathrm{Mn},{ }^{40} \mathrm{~K},{ }^{232} \mathrm{Th},{ }^{235} \mathrm{U}\right.$, $\left.{ }^{238} \mathrm{U}\right)$. The bulk assumed composition is dust (77\%) plus amorphous water ice (23\%), the relative mass fractions being derived from the input $10 \%$ porosity. Various initial configurations have been simulated with different formation delays (from 1 to over $10 \mathrm{Myr}$ ) including different amounts of short-lived radiogenic elements (with the case no short-lived elements at all) to evaluate the differentiation state of Orcus. Our simulations show that a layer of amorphous water ice of a few kilometers thick would be maintained at the surface of the body, while the remaining of the volume is occupied by crystalline water ice. For all cases we considered, the heating induced by radiogenic elements leads to the melting of the core. This effect is strengthened if ammonia is present inside the body, because it reduces the melting point temperature of the mixture and allows for the production of a liquid phase even under moderate heating. This liquid phase could easily reach layers close to the surface through cracks in the ice matrix (if not directly produced in subsurface layers in the more extreme cases), and therefore make Orcus and intermediate to large water-bearing KBOs the subject of potential astrobiological interest.

As a consequence, even if it is premature to suggest that Orcus did undergo a recent event of cryovolcanism, our results show that this event did take place at some point of Orcus' history and most probably supplied crystalline water ice to the surface. Recent laboratory experiments by Zheng et al. (2009) show that the ion-irradiation-induced amorphization of crystalline water ice on a KBO at $\sim 40 \mathrm{~K}$ can be incomplete and be efficiently balanced by re-crystallisation (starting at $50 \mathrm{~K}$ ), so that crystalline water ice can survive and still be detectable at the surface over timescales longer than the Solar System age. Both from the latter results and our model, we can envision that some of the crystalline ice we currently observe at the surface of Orcus is actually the remnant of a past cryovolcanic event. In addition, in the most probable case of our simulations, a large volume of volatiles are currently present in Orcus subsurface layer, awaiting to be revealed by future surface alterations.

Acknowledgements. We thank D. Jewitt for fruitful suggestions and are grateful to J.C. Cook for kindly sharing with us estimated optical constants of ammonium, to S. Fornasier et al. for providing us with their Orcus visible spectra, and to S. Spjuth for the computation of the visible geometric albedo of Charon. This material is based upon work supported by the National Aeronautics and Space Administration through the NASA Astrobiology Institute under Cooperative Agreement No. NNA04CC08A issued through the Office of Space Science. B. Yang acknowledges support from a NASA Origins grant and A. Guilbert-Lepoutre from a NASA Herschel grant, both to D. Jewitt. Part of the data presented here were obtained at the W.M. Keck Observatory, which is operated as a scientific partnership among the California Institute of Technology, the University of California and NASA. Based on observations obtained at the Gemini Observatory, which is operated by the Association of Universities for Research in Astronomy, Inc., under a cooperative agreement with the NSF on behalf of the Gemini partnership. The authors wish to recognize and acknowledge the very significant cultural role and reverence that the summit of Mauna Kea has always had within the indigenous Hawaiian community.

\section{References}

Baratta, G. A., Domingo, M., Ferini, G., et al. 2003, Nuclear Instruments and Methods in Physics Research B, 209, 283

Barkume, K. M., Brown, M. E., \& Schaller, E. L. 2008, AJ, 135, 55
Barucci, M. A., Cruikshank, D. P., Dotto, E., et al. 2005, A\&A, 439, L1 Barucci, M. A., Merlin, F., Guilbert, A., et al. 2008, A\&A, 479, L13 Bauer, J. M., Roush, T. L., Geballe, T. R., et al. 2002, Icarus, 158, 178 Brown, M., Ragozzine, D., Stansberry, J., \& Fraser, W. 2010, AJ, submitted Brown, M. E. 2008, The Largest Kuiper Belt Objects, ed. M. A. Barucci,

H. Boehnhardt, D. P. Cruikshank, \& A. Morbidelli, 335

Brown, M. E., Barkume, K. M., Blake, G. A., et al. 2007a, AJ, 133, 284 Brown, M. E., Barkume, K. M., Ragozzine, D., \& Schaller, E. L. 2007b, Nature, 446, 294

Brown, M. E., Rabinowitz, D. L., Trujillo, C. A., et al. 2004, IAU Circ., 8291, 1 Brown, M. E., \& Trujillo, C. A. 2004, AJ, 127, 2413

Brown, M. E., Trujillo, C. A., \& Rabinowitz, D. L. 2005, ApJ, 635, L97 Brucker, M. J., Grundy, W. M., Stansberry, J. A., et al. 2009, Icarus, 201, 284 Brunetto, R., Barucci, M. A., Dotto, E., \& Strazzulla, G. 2006, ApJ, 644, 646 Buie, M. W., Grundy, W. M., Young, E. F., Young, L. A., \& Stern, S. A. 2010, AJ, 139, 1117

Canup, R. M. 2005, Science, 307, 546

Castillo-Rogez, J. C., Matson, D. L., Sotin, C., et al. 2007, Icarus, 190, 179

Cook, J. C., Desch, S. J., Roush, T. L., Trujillo, C. A., \& Geballe, T. R. 2007, ApJ, 663, 1406

Cook, J. C., Olkin, C. B., Desch, S. J., et al. 2009, in Lunar and Planetary Inst. Technical Report, Lunar and Planetary Institute Science Conference Abstracts, 40, 2222

Cooper, J. F., Christian, E. R., Richardson, J. D., \& Wang, C. 2003, Earth Moon and Planets, 92, 261

Dalle Ore, C. M., Barucci, M. A., Emery, J. P., et al. 2009, A\&A, 501, 349

de Bergh, C., Delsanti, A., Tozzi, G. P., et al. 2005, A\&A, 437, 1115

Delsanti, A., Hainaut, O., Jourdeuil, E., et al. 2004, A\&A, 417, 1145

Delsanti, A. C., Boehnhardt, H., Barrera, L., et al. 2001, A\&A, 380, 347

Desch, S. J., Cook, J. C., Doggett, T. C., \& Porter, S. B. 2009, Icarus, 202, 694

Duffard, R., Ortiz, J. L., Thirouin, A., Santos-Sanz, P., \& Morales, N. 2009, A\&A, 505, 1283

Durham, W. B., McKinnon, W. B., \& Stern, L. A. 2005, Geophys. Res. Lett., 32, 18202

Fornasier, S., Barucci, M. A., de Bergh, C., et al. 2009, A\&A, 508, 457

Fornasier, S., Dotto, E., Barucci, M. A., \& Barbieri, C. 2004, A\&A, 422, L43

Fraser, W., \& Brown, M. E. 2009, in AAS/Division for Planetary Sciences Meeting Abstracts, AAS/Division for Planetary Sciences Meeting Abstracts, $41,65.03$

Gladman, B., Marsden, B. G., \& Vanlaerhoven, C. 2008, Nomenclature in the Outer Solar System, ed. M. A. Barucci, H. Boehnhardt, D. P. Cruikshank, \& A. Morbidelli, 43

Gomes, R. S. 2003, Icarus, 161, 404

Grundy, W. M., \& Schmitt, B. 1998, J. Geophys. Res., 103, 25809

Guilbert, A., Alvarez-Candal, A., Merlin, F., et al. 2009, Icarus, 201, 272

Guilbert-Lepoutre, A., Lasue, J., Federico, C., Coradini, A., \& Orosei, R. 2010, New 3D Thermal Evolution Model for Small Icy Bodies. Application to Trans-Neptunian Objects, under revision for A\&A

Hapke, B. 1981, J. Geophys. Res., 86, 4571

Hapke, B. 1993, Theory of reflectance and emittance spectroscopy, ed. B. Hapke Jewitt, D. C. 2002, AJ, 123, 1039

Jewitt, D. C., \& Luu, J. 2004, Nature, 432, 731

Kargel, J. S. 1992, Icarus, 100, 556

Kargel, J. S. 1995, Earth Moon and Planets, 67, 101

Kargel, J. S., \& Strom, R. G. 1990, in Lunar and Planetary Inst. Technical Report, Lunar and Planetary Institute Science Conference Abstracts, 21, 599

Kenyon, S. J., Bromley, B. C., O’Brien, D. P., \& Davis, D. R. 2008, Formation and Collisional Evolution of Kuiper Belt Objects, ed. M. A. Barucci, H. Boehnhardt, D. P. Cruikshank, \& A. Morbidelli, 293

Khare, B. N., Sagan, C., Arakawa, E. T., et al. 1984, Icarus, 60, 127

Klinger, J. 1981, Icarus, 47, 320

Lewis, J. S. 1972, Icarus, 16, 241

Malhotra, R. 1993, Nature, 365, 819

Malhotra, R. 1995, AJ, 110, 420

McKinnon, W. B., Simonelli, D. P., \& Schubert, G. 1997, Composition, Internal Structure, and Thermal Evolution of Pluto and Charon, ed. Stern, S. A., \& Tholen, D. J., 295

Merlin, F., Guilbert, A., Dumas, C., et al. 2007, A\&A, 466, 1185

Merlin, F., Alvarez-Candal, A., Delsanti, A., et al. 2009, AJ, 137, 315

Merlin, F., Barucci, M., de Bergh, C., et al. 2010a, Chemical and physical properties of the variegated Pluto and Charon surfaces, Icarus, in Press

Merlin, F., Barucci, M., de Bergh, C., et al. 2010b, Icarus, 208, 945

Moore, M. H., Ferrante, R. F., Hudson, R. L., \& Stone, J. N. 2007, Icarus, 190, 260

Moore, M. H., \& Hudson, R. L. 2003, Icarus, 161, 486

Noll, K. S., Grundy, W. M., Chiang, E. I., Margot, J., \& Kern, S. D. 2008, Binaries in the Kuiper Belt, ed. M. A. Barucci, H. Boehnhardt, D. P. Cruikshank, \& A. Morbidelli, 345 
Oke, J. B., Cohen, J. G., Carr, M., et al. 1995, PASP, 107, 375

Perets, H. B., \& Naoz, S. 2009, ApJ, 699, L17

Pinilla-Alonso, N., Licandro, J., Gil-Hutton, R., \& Brunetto, R. 2007, A\&A, 468, L25

Pinilla-Alonso, N., Licandro, J., \& Lorenzi, V. 2008, A\&A, 489, 455

Pinilla-Alonso, N., Brunetto, R., Licandro, J., et al. 2009, A\&A, 496, 547

Prialnik, D., Benkhoff, J., \& Podolak, M. 2004, Modeling the structure and activity of comet nuclei, ed. M. C. Festou, H. U. Keller, \& H. A. Weaver, 359

Quirico, E., \& Schmitt, B. 1997, Icarus, 127, 354

Rabinowitz, D. L., Barkume, K., Brown, M. E., et al. 2006, ApJ, 639, 1238

Rabinowitz, D. L., Schaefer, B. E., \& Tourtellotte, S. W. 2007, AJ, 133, 26

Rabinowitz, D. L., Schaefer, B. E., Schaefer, M., \& Tourtellotte, S. W. 2008, AJ, 136,1502

Schaller, E. L., \& Brown, M. E. 2007a, ApJ, 670, L49

Schaller, E. L., \& Brown, M. E. 2007b, ApJ, 659, L61

Schmitt, B., Espinasse, S., Grim, J. A., Greenberg, J. M., \& Klinger, J. 1989, in ESA SP-302: Physics and Mechanics of Cometary Materials, ed. I. J. J. Hunt, \& E. T. D. Guyenne, 65

Schmitt, B., de Bergh, C., \& Festou, M. 1998, The Observatory, 118, 312

Sheppard, S. S. 2007, AJ, 134, 787

Sicardy, B., Bellucci, A., Gendron, E., et al. 2006, Nature, 439, 52
Snodgrass, C., Carry, B., Dumas, C., \& Hainaut, O. 2009, 2010, A\&A, 511, A72 Stansberry, J., Grundy, W., Brown, M., et al. 2008, Physical Properties of Kuiper Belt and Centaur Objects: Constraints from the Spitzer Space Telescope, ed. M. A. Barucci, H. Boehnhardt, D. P. Cruikshank, \& A. Morbidelli (Tucson: University of Arizona Press), 161

Stevenson, D. J. 1982, Nature, 298, 142

Strazzulla, G., Leto, G., Baratta, G. A., \& Spinella, F. 1991, J. Geophys. Res., 96,17547

Tody, D. 1986, in SPIE Conf. Ser., ed. D. L. Crawford, 627, 733

Trujillo, C. A., Brown, M. E., Rabinowitz, D. L., \& Geballe, T. R. 2005, ApJ, 627,1057

Verbiscer, A., \& Helfenstein, P. 1998, in Solar System Ices, ed. B. Schmitt, C. de Bergh, \& M. Festou, A\&SS Library, 227, 157

Weidenschilling, S. J. 2004, From icy grains to comets, ed. M. C. Festou, H. U. Keller, \& Weaver, H. A., 97

Wong, M. H., Lunine, J. I., Atreya, S. K., et al. 2008, Reviews in Mineralogy and Geochemistry, 68, 219

Zheng, W., Jewitt, D., \& Kaiser, R. 2009, J. Phys. Chem A, 113, 11174

Zubko, V. G., Mennella, V., Colangeli, L., \& Bussoletti, E. 1996, MNRAS, 282, 1321 